\title{
Strong solution for a stochastic model of two-dimensional second grade fluids : existence, uniqueness and asymptotic behaviour
}

\author{
Paul André Razafimandimby ${ }^{1,2}$ and Mamadou Sango ${ }^{1}$ \\ ${ }^{1}$ Department of Mathematics and Applied Mathematics, University of Pretoria, \\ Pretoria 0002, South Africa \\ ${ }^{2}$ Mathematics section, The Abdus Salam International Centre of Theoretical \\ Physics, Strada Costiera, 11, I - 34151 Trieste, Italy
}

\begin{abstract}
We investigate a stochastic evolution equation for the motion of a second grade fluid filling a bounded domain of $\mathbb{R}^{2}$. Global existence and uniqueness of strong probabilistic solution is established. In contrast to previous results on this model we show that the sequence of Galerkin approximation converges in mean square to the exact strong probabilistic solution of the problem. We also give two results on the long time behaviour of the solution. Mainly we prove that the strong solution of our stochastic model converges exponentially in mean square to the stationary solution of the time-independent second grade fluids equations if the deterministic part of the external force does not depend on time. If the deterministic forcing term explicitly depends on time, then the strong probabilistic solution decays exponentially in mean square.
\end{abstract}

\section{Introduction}

One of the most challenging problems in applied sciences is the turbulence in hydrodynamic. On basis of Reynolds' work, it was postulated that hydrodynamic turbulence is composed of slow (deterministic) and fast (stochastic) oscillating components. For this reason the idea of introducing a noise term for modeling a turbulent evolutive fluid has now become widely recognized. Such approach in the mathematical investigation toward the understanding of the Newtonian turbulence phenomenon was pioneered by Bensoussan and Temam in [4] where they studied the Stochastic Navier-Stokes Equation (SNSE). Since then stochastic partial differential equations and stochastic models of Newtonian fluid dynamics have been the object of intense investigations which have generated several important results. We refer for instance to [3], [2], [10], [11], [20], [21], [22], [39], [33],[46],[44], [45]. Similar investigations for stochastic models of Non-Newtonian fluids have almost not been undertaken except in very few works. We refer, for instance, to [7], [29], [30], [31], [41], [42], [43] for some relevant results in that direction. It should be noted that the models investigated in the first four papers occur very naturally from the kinetic theory of polymer dynamics. Indeed they arise from the reformulation of Fokker-Planck or diffusion equations as stochastic differential equations (see [37]).

In the present work we are interested in the mathematical analysis of the stochastic model for the incompressible second grade fluid which is a particular class of NonNewtonian fluid. More precisely, we assume that a connected and bounded open set $\mathcal{O}$ of 
$\mathbb{R}^{2}$ with boundary $\partial \mathcal{O}$ of class $\mathcal{C}^{3}$, a finite time horizon $[0, T]$, and an initial value $u_{0}$ are given. We consider the problem

$$
\left\{\begin{array}{l}
d(u-\alpha \Delta u)+(-\nu \Delta u+\operatorname{curl}(u-\alpha \Delta u) \times u+\nabla \mathfrak{P}) d t=F(u, t) d t+G(u, t) d W \\
\operatorname{in} \mathcal{O} \times(0, T] \\
\operatorname{div} u=0 \text { in } \mathcal{O} \times(0, T], \\
u=0 \text { in } \partial \mathcal{O} \times(0, T], \\
u(0)=u_{0} \text { in } \mathcal{O}
\end{array}\right.
$$

where $u=\left(u_{1}, u_{2}\right)$ and $\mathfrak{P}$ represent the random velocity and modified pressure, respectively. The system is to be understood in the Itô sense. It is the equation of motion of an incompressible second grade fluid driven by external forces $F(u, t)$ and $G(u, t) d W$, where $W$ is a $m$-dimensional standard Wiener process defined on a prescribed complete probability space $(\Omega, \mathcal{F}, P)$. Here $m \geq 1$ is a fixed natural number. We refer to [34], [23], [24] and [25] for a comprehensive theory of the second grade fluids.

The interest in the investigation of the second grade fluids arises from the fact that it is an admissible model of a large class Non-Newtonian fluids such as industrial fluids, slurries, polymer melts,.... Furthermore, "second grade fluid compatible with thermodynamics has general and pleasant properties such as boundedness, stability, and exponential decay" (see [23]). It can also be taken as a generalization of the Navier-Stokes Equation (NSE). Indeed they reduce to NSE when $\alpha=0$. Moreover, recent work [28] shows that it is a good approximation of the NSE. We also refer to [12], [27],[26], [48], [47] for interesting discussions about their relationship with other fluid models. Due to these nice properties, the mathematical analysis of the second grade fluid has attracted many prominent researchers in the deterministic case. The pioneering mathematical work on nonlinear fluids such as acqueous solutions polymers was undertaken by the Soviet mathematician Oskolkov in [35] and [36]. This work influenced the emergence of the first relevant analysis of the equations for the second grade fluids in the joint work of Cioranescu and Ouazar published in [17] and [18]. Their method was based on a priori estimates for the Galerkin solution using a special basis consisting of eigenfunctions corresponding to the scalar product associated to the operator $\operatorname{curl}(u-\alpha \Delta u)$. They proved global existence and uniqueness without restriction on the initial data for the two dimensional case. Bernard in [5], Cioranescu and Girault in [16] extended their results to the three dimensional case; global existence was obtained with some reasonable restrictions on the initial data.

To the best of our knowledge the system (1) was only analyzed in [41] and [43] where the authors focused on proving the existence of weak probabilistic solutions and the behaviour of the solution as $\alpha \rightarrow 0$, respectively. The existence in [41] was achieved by using Galerkin method combined with some deep compactness results due to Prokhorov and Skorokhod. The main goals of the present work are the following:

- Prove the existence and uniqueness results for the strong probabilistic solution of the stochastic second grade fluids (see Theorem 3.4 and 4.1). In contrast to [41], here we do not use Prokhorov's and Skorokhod's results.

- Show that, in contrast to [41] again, the whole sequence of Galerkin approximation converges to the strong probabilistic solution in mean square (see Theorem 4.2).

- Analyze the long time behaviour of the strong probabilistic solution. Mainly, we show that under some additional hypotheses on the data it decays exponentially in mean square (see Theorem 5.2). We also prove that if the deterministic part of the 
external forces is time independent then the strong solution of our stochastic model converges exponentially in mean square to the stationary solution (see Theorem 5.6).

To prove the results in the first two points we use a method which was developed by Breckner for the first time in [8] and used later on in [14]. For the third point we mainly follow the idea used in [13] and [15]. However, the problem we treat here do not fall into the general framework considered in $[8,14,13,15]$. It should be noted that solving (1) is rather challenging even in the deterministic case, the nature of the nonlinearities being one of the main difficulties in addition to the complex structure of the equations. Indeed (1) is a highly nonlinear partial differential equations (PDEs) which do not contain any smoothing term like many nonlinear evolution equations such as the Navier-Stokes equations. The linear term $-\Delta u$ is not smoothing, it is rather acting like a damping term. Beside this, the introduction of the noise term $G(u, t) d W$ in the stochastic version induces the appearance of some expressions that are very hard to control when proving crucial estimates. Overcoming these problems will require a tour de force in the work.

The present work, [41], and [43] could by no means exhaust the mathematical analysis of the problem. We hope that they will stimulate further research in this field, where many questions still stand opened.

To close this introduction we outline the organization of the article. In addition to the introduction the paper consists of four other sections. In section 2 we give some notations, necessary backgrounds of probabilistic or analytical nature. We formulate and prove our existence result by means of Galerkin method in Section 3. The uniqueness result and the convergence of the whole sequence of Galerkin solution are stated and proved in the fourth section of the paper. In the last section we analyze the asymptotic long time behaviour of the solution of our model.

\section{Notations-Preliminaries}

Let us start with some informations about functional spaces needed in this work. Let $\mathcal{O}$ be an open subset of $\mathbb{R}^{n}$, let $1 \leq p \leq \infty$ and let $k$ be a nonnegative integer. We consider the well-known Lebesgue and Sobolev spaces $L^{p}(\mathcal{O})$ and $W^{k, p}(\mathcal{O})$, respectively. When $p=2$, we write $W^{k, 2}(\mathcal{O})=H^{k}(\mathcal{O})$. We denote by $W_{0}^{k, p}(\mathcal{O})$ the closure in $W^{k, p}(\mathcal{O})$ of $C_{c}^{\infty}(\mathcal{O})$ the space of infinitely differentiable function with compact support in $\mathcal{O}$. If $p=2$, we denote $W_{0}^{k, p}(\mathcal{O})$ by $H_{0}^{k}(\mathcal{O})$. We assume that the Hilbert space $H_{0}^{1}(\mathcal{O})$ is endowed with the scalar product

$$
((u, v))=\int_{D} \nabla u . \nabla v d x=\sum_{i=1}^{n} \int_{D} \frac{\partial u}{\partial x_{i}} \frac{\partial v}{\partial x_{i}} d x
$$

where $\nabla$ is the gradient operator. The norm $\|$.$\| generated by this scalar product is$ equivalent to the usual norm of $W^{1,2}(D)$ in $H_{0}^{1}(\mathcal{O})$. If the domain $D$ is smooth enough (of class $\mathcal{C}^{m}$ for instance), then for any $m$ and $p$ such that $m p>n$ the embedding

$$
W^{j+m, p}(\mathcal{O}) \subset W^{j, q}(\mathcal{O})
$$

is compact for any $1 \leq q \leq \infty$. More general embedding theorems can be found in [1] and references therein.

Next we define some probabilistic evolution spaces necessary throughout the paper. Let $\left(\Omega, \mathcal{F},\left(\mathcal{F}_{t}\right)_{0 \leq t \leq T}, P\right)$ be a given stochastic basis; that is, $(\Omega, \mathcal{F}, P)$ is complete probability space and $\left(\mathcal{F}_{t}\right)_{0 \leq t \leq T}$ is an increasing sub- $\sigma$-algebras of $\mathcal{F}$ such that $\mathcal{F}_{0}$ contains every $P$-null subset of $\Omega$. For any real Banach space $X$ endowed with the norm $\|\cdot\|_{X}$, for any 
$p \geq 1$, we denote by $L^{p}(0, T ; X)$ the space of $X$-valued measurable functions $u$ defined on $[0, T]$ such that

$$
\|u\|_{L^{p}(0, T ; X)}=\left(\int_{0}^{T}\|u\|_{X}^{p} d t\right)^{\frac{1}{p}}<\infty .
$$

For any $r, p \geq 1$ let $L^{p}\left(\Omega, \mathcal{F}, P ; L^{r}(0, T ; X)\right)$ be the space of processes $u=u(\omega, t)$ with values in $X$ defined on $\Omega \times[0, T]$ such that

1. $u$ is measurable with respect to $(\omega, t)$ and for each $t, u(., t)$ is $\mathcal{F}^{t}$-measurable.

2. $u(t, \omega) \in X$ for almost all $(\omega, t)$ and

$$
\|u\|_{L^{p}\left(\Omega, \mathcal{F}, P ; L^{r}(0, T ; X)\right)}=\left(E\left(\int_{0}^{T}\|u\|_{X}^{r} d t\right)^{\frac{p}{r}}\right)^{\frac{1}{p}}<\infty
$$

where $E$ denotes the mathematical expectation with respect to the probability measure $P$.

When $r=\infty$, we write

$$
\|u\|_{L^{p}\left(\Omega, \mathcal{F}, P ; L^{\infty}(0, T ; X)\right)}=\left(E \sup _{0 \leq t \leq T}\|u\|_{X}^{p} d t\right)^{\frac{1}{p}}<\infty .
$$

We proceed with the definitions of some additional spaces frequently used in this work. In what follows we denote by $\mathbb{X}$ the space of $\mathbb{R}^{2}$-valued functions such that each component belongs to $X$. A simply-connected and bounded domain $\mathcal{O} \subset \mathbb{R}^{2}$ with boundary $\partial \mathcal{O}$ of class $\mathcal{C}^{3}$ is given. We introduce the spaces

$$
\begin{aligned}
\mathcal{V} & =\left\{u \in \mathcal{C}_{c}^{\infty}(\mathcal{O}) \text { such that } \operatorname{div} u=0\right\} \\
\mathbb{V} & =\text { closure of } \mathcal{V} \text { in } \mathbb{H}^{1}(\mathcal{O}) \\
\mathbb{H} & =\text { closure of } \mathcal{V} \text { in } \mathbb{L}^{2}(\mathcal{O})
\end{aligned}
$$

We denote by $(\cdot, \cdot)$ and $|\cdot|$ the inner product in $\mathbb{L}^{2}(\mathcal{O})$ (in $\left.\mathbb{H}\right)$ and the induced norm, respectively. The inner product and the norm of $\mathbb{H}_{0}^{1}(\mathcal{O})$ are denoted respectively by $((.,)$. and $\|\cdot\|$. We endow the space $\mathbb{V}$ with the norm generated by the following scalar product

$$
(u, v)_{\mathbb{V}}=(u, v)+\alpha((u, v)), \text { for any } u \text { and } v \in \mathbb{V} \text {; }
$$

this norm turns out to be equivalent to $\|\cdot\|$. More precisely, the following inequalities hold

$$
\left(\mathcal{P}^{2}+\alpha\right)^{-1}|v|_{\mathbb{V}}^{2} \leq\|v\|^{2} \leq(\alpha)^{-1}|v|_{\mathbb{V}}^{2} \text {, for any } v \in \mathbb{V},
$$

where $\mathcal{P}$ is the constant from Poincaré's inequality.

We also introduce the following space

$$
\mathbb{W}=\left\{u \in \mathbb{V} \text { such that } \operatorname{curl}(u-\alpha \Delta u) \in L^{2}(\mathcal{O})\right\} .
$$

It is convenient to recall a very important result from [17] concerning the space $\mathbb{W}$. 
Lemma 2.1. The following (algebraic and topological) identity holds

$$
\mathbb{W}=\widehat{\mathbb{W}},
$$

where

$$
\widehat{\mathbb{W}}=\left\{v \in \mathbb{H}^{3}(\mathcal{O}) \text { such that } \operatorname{div} v=0 \text { and } v_{\mid \partial D}=0\right\} .
$$

Moreover, there exists a positive constant $C$ such that

$$
|v|_{\mathbb{H}^{3}(\mathcal{O})}^{2} \leq C\left(|v|_{\mathbb{V}}^{2}+|\operatorname{curl}(v-\alpha \Delta)|^{2}\right)
$$

for any $v \in \widehat{\mathbb{W}}$.

Thanks to this lemma we can endow the space $\mathbb{W}$ with the scalar product

$$
(u, v)_{\mathbb{W}}=(u, v)_{\mathbb{V}}+(\operatorname{curl}(u-\alpha \Delta u), \operatorname{curl}(v-\alpha \Delta v)) .
$$

From now on, we identify the space $\mathbb{V}$ with its dual space $\mathbb{V}^{\star}$ via the Riesz representation, and we have the Gelfand triple

$$
\mathbb{W} \subset \mathbb{V} \subset \mathbb{W}^{\star},
$$

where each space is dense in the next one and the inclusions are continuous. We denote by $\langle f, v\rangle$ the action of any element $f$ of $\mathbb{W}^{*}$ on an element $v \in \mathbb{W}$. It follows from the above identification that we can write

$$
(v, w)_{\mathbb{V}}=\langle v, w\rangle,
$$

for any $(v, w) \in \mathbb{V} \times \mathbb{W}$.

Let us state an important theorem on solvability of "generalized Stokes equations". Its proof can be derived from an adaptation of the results obtained by Solonnikov in [50, 51].

Theorem 2.2. Let $\mathcal{O}$ be a connected, bounded open set in $\mathbb{R}^{2}$ of class $\mathcal{C}^{l}$ (resp., Lipschitz domain) and let $f$ be a function in $\mathbb{H}^{l}, l \in \mathbb{N} \backslash\{0\}($ resp., $l=0)$. The system

$$
\left\{\begin{array}{l}
v-\alpha \Delta v+\nabla q=f \text { in } \mathcal{O} \\
\operatorname{div} v=0 \text { in } \mathcal{O} \\
v=0 \text { on } \partial \mathcal{O}
\end{array}\right.
$$

has a solution $v \in \mathbb{H}^{l+2} \cap \mathbb{V}$. If $f \in \mathbb{H}^{l} \cap \mathbb{H}$ then $v$ is unique and the following relations hold

$$
\begin{aligned}
(v, g)_{\mathbb{V}} & =(f, g), \\
|v|_{\mathbb{H}^{l+2}} & \leq C|f|_{\mathbb{H}},
\end{aligned}
$$

for any $g \in \mathbb{V}$.

Now we turn our attention to the definition of some operators needed in this work. We denote by $\mathbb{P}: \mathbb{L}^{2}(\mathcal{O}) \rightarrow \mathbb{H}$ the usual Helmholtz-Leray projector. We also introduce the well known Stokes operator $A=-\mathbb{P} \Delta$ with domain $D(A)=\mathbb{H}^{2}(\mathcal{O}) \cap \mathbb{V}$. It follows from the Theorem 2.2 that the operator $(I+\alpha A)^{-1}$ defines an isomorphism from $\mathbb{H}^{l}(\mathcal{O}) \cap \mathbb{H}$ into $\mathbb{H}^{l+2}(\mathcal{O}) \cap \mathbb{V}$ provided that $\mathcal{O}$ is of class $\mathcal{C}^{l}, l \geq 1$. Moreover, the following properties hold

$$
\begin{gathered}
\left((I+\alpha A)^{-1} f, v\right)_{\mathbb{V}}=(f, v), \\
\left|(I+\alpha A)^{-1} f\right|_{\mathbb{V}} \leq C|f|,
\end{gathered}
$$


for any $f \in \mathbb{H}^{l}(\mathcal{O}) \cap \mathbb{V}$ and any $v \in \mathbb{V}$. From these facts, $\widehat{A}=(I+\alpha A)^{-1} A$ defines a continuous linear operator from $\mathbb{H}^{l}(\mathcal{O}) \cap \mathbb{V}$ onto itself for $l \geq 2$. $\widehat{A}$ satisfies

$$
(\widehat{A} u, v)_{\mathbb{V}}=(A u, v)=((u, v)),
$$

for any $u \in \mathbb{W}$ and $v \in \mathbb{V}$. Clearly,

$$
(\widehat{A} u, u)_{\mathbb{V}}=\|u\|^{2}
$$

for any $u \in \mathbb{W}$.

Following the notation of the Navier-Stokes Equations we set

$$
b(u, v, w)=\sum_{i, j=1}^{2} \int_{D} u_{i} \frac{\partial v_{j}}{\partial x_{i}} w_{j} d x,
$$

for any $u, v, w \in \mathcal{V}$. We state the following identity (see for instance [5] and [17]):

$$
((\operatorname{curl} \Phi) \times v, w)=b(v, \Phi, w)-b(w, \Phi, v),
$$

for any smooth (solenoidal) functions $\Phi, v$ and $w$. Now we recall the following inequalities whose proof can be found in [5], [17] (see also [40])

Lemma 2.3. For any $u \in \mathbb{W}, v \in \mathbb{W}$ and $w \in \mathbb{W}$ we have

$$
|(\operatorname{curl}(u-\alpha \Delta u) \times v, w)| \leq C|u|_{\mathbb{H}^{3}}|v|_{\mathbb{V}}|w|_{\mathbb{W}} .
$$

We also have

$$
|(\operatorname{curl}(u-\alpha \Delta u) \times u, w)| \leq C|u|_{\mathbb{V}}^{2}|w|_{\mathbb{W}},
$$

for any $u \in \mathbb{W}$ and $w \in \mathbb{W}$.

We have a very important consequence of this lemma.

Lemma 2.4. There exists a bilinear operator $\widehat{B}: \mathbb{W} \times \mathbb{V} \rightarrow \mathbb{W}^{*}$ such that

$$
<\widehat{B}(u, v), w>=(\mathbf{P}(\operatorname{curl}(u-\alpha \Delta u) \times v), w), \text { for any }(u, v, w) \in \mathbb{W} \times \mathbb{V} \times \mathbb{W},
$$

For any $u \in \mathbb{W}$ and $v \in \mathbb{V}$ there holds

$$
|\widehat{B}(u, v)|_{\mathbb{W}^{*}} \leq C|u|_{\mathbb{W}}|v|_{\mathbb{V}}
$$

If $u=v \in \mathbb{W}$ in the above estimate, then

$$
|\widehat{B}(u, u)|_{\mathbb{W} *} \leq C_{B}|u|_{\mathbb{V}}^{2} .
$$

In addition

$$
<\widehat{B}(u, v), v>=0
$$

which in turn implies

$$
<\widehat{B}(u, v), w>=-<\widehat{B}(u, w), v>
$$

for any $u \in \mathbb{W}, v \in \mathbb{V}$ and $w \in \mathbb{W}$.

Proof. First, note that the right hand side of (12) defines a trilinear form on $\mathbb{W} \times \mathbb{V} \times \mathbb{W}$. Thanks to the equivalence of the norm $|\cdot|_{\mathbb{H}^{3} \mathcal{O}}$ and the norm $|.| \mathbb{W}$ on $\mathbb{W}$ and the fact that $\boldsymbol{P}$ is an self-adjoint operator, inequality (10) induces the existence of $\widehat{B}(u, v) \in \mathbb{W}^{*}$ which satisfies (12). The inequalities (13) and (14) follows from (10) and (11) respectively. The identity (16) follows from (15), which in turn can be checked by using (9) with $\Phi=$ $\operatorname{curl}(u-\alpha \Delta u)$ and $w=v$. 


\section{$3 \quad$ Existence of the strong probabilistic solution}

In this part we investigate the existence of the strong probabilistic solution of problem (1). This section contains two subsections. The first one is devoted to the formulation of the hypotheses and the statement of the existence theorem while the second consists of its proof.

\subsection{Hypotheses and statement of the existence theorem}

We start by stating some hypotheses relevant for most part of the paper. First, we shall assume that throughout we endow the prescribed complete probability space $(\Omega, \mathcal{F}, P)$ with the filtration $\mathcal{F}^{t}, 0 \leq t \leq T$, which is the $\sigma$-field generated by the random variables $\{W(s), 0 \leq s \leq t\}$ and the null sets of $\mathcal{F}$. The functions $F$ and $G$ introduced in (1) induce the following mappings denoted by the same symbols.

(F) The $\mathbb{V}$-valued mapping $F$ defined on $\mathbb{V} \times[0, T]$ is measurable in the second variable and we assume that

$$
F(0, t)=0 \text {, for any } t \in[0, T]
$$

and

$$
\left|F\left(u_{1}, t\right)-F\left(u_{2}, t\right)\right|_{\mathbb{V}} \leq C\left|u_{1}-u_{2}\right|_{\mathbb{V}}
$$

for any $t \in[0, T]$ and for any $\left(u_{1}, u_{2}\right) \in \mathbb{V} \times \mathbb{V}$.

For any Banach space $X$ we set

$$
X^{\otimes m}=\underbrace{X \times \cdots \times X}_{m \text { times }} .
$$

(G) The mapping $G: \mathbb{V} \times[0, T] \rightarrow \mathbb{V}^{\otimes m}$ is measurable with respect to its second variable. Furthermore, we suppose that

$$
G(0, t)=0, \text { for any } t \in[0, T],
$$

and

$$
\left|G\left(u_{1}, t\right)-G\left(u_{2}, t\right)\right|_{\mathbb{V} \otimes m} \leq C\left|u_{1}-u_{2}\right|_{\mathbb{V}},
$$

for any $t \in[0, T]$ and for any $u_{1}, u_{2} \in \mathbb{V}$.

We can define on $\mathbb{V} \times[0, T]$ two operators $\widehat{F}$ and $\widehat{G}$ taking values in $\mathbb{W}$ and $\mathbb{W}^{\otimes m}$, respectively, by setting

$$
\widehat{F}(u, t)=(I+\alpha A)^{-1} F(u, t),
$$

and

$$
\widehat{G}(u, t)=(I+\alpha A)^{-1} G(u, t) .
$$

Thanks to the features of $(I+\alpha A)^{-1}$ all the properties such as measurability in $t$ of $F$ and $G$ are verified by $\widehat{F}$ and $\widehat{G}$. In particular, we have

$$
\begin{aligned}
\left|\widehat{F}\left(u_{1}, t\right)-\widehat{F}\left(u_{2}, t\right)\right|_{\mathbb{V}} & \leq C_{F}\left|u_{1}-u_{2}\right|_{\mathbb{V}}, \\
\left|\widehat{G}\left(u_{1}, t\right)-\widehat{G}\left(u_{2}, t\right)\right|_{\mathbb{V} \otimes m} & \leq C_{G}\left|u_{1}-u_{2}\right|_{\mathbb{V}} .
\end{aligned}
$$


Remark 3.1. The condition (17) is required without loss of generality in order to simplify the computations. We can assume that $F(0, t) \neq 0$, but then

$$
\int_{0}^{T}|F(0, t)|_{\mathbb{V}}^{p} d t<\infty
$$

should hold for any $2 \leq p<\infty$. The same remark applies to the operator $G$.

Alongside (1), we consider the abstract evolution stochastic problem

$$
\left\{\begin{array}{l}
d u(t)+\nu \widehat{A} u(t) d t+\widehat{B}(u(t), u(t)) d t=\widehat{F}(u(t), t) d t+\widehat{G}(u(t), t) d W(t), \\
u_{0}=u(0),
\end{array}\right.
$$

which holds in $\mathbb{W}^{*}$. With the properties of the operators involved we can prove stochastic process $u$ satisfies (23) if and only if it verifies (1) in the weak sense of partial differential equations.

Now we introduce the concept of solution of the problem (1) relevant here.

Definition 3.2. By a strong probabilistic solution of the system (1), we mean a stochastic process $u$ such that

1. $u \in L^{p}\left(\Omega, \mathcal{F}, P ; L^{\infty}(0, T ; \mathbb{W})\right), 2 \leq p<\infty$.

2. For all $t, u(t)$ is $\mathcal{F}^{t}$-measurable,

3. $P$-a.s the following integral identity holds

$$
\begin{aligned}
& (u(t)-u(0), v)_{\mathbb{V}}+\int_{0}^{t}[\nu((u, v))+(\operatorname{curl}(u(s)-\alpha \Delta u(s)) \times u, v)] d s \\
& =\int_{0}^{t}(F(u(s), s), v) d s+\int_{0}^{t}(G(u(s), s), v) d W(s),
\end{aligned}
$$

for any $t \in(0, T]$ and $v \in \mathbb{W}$. Or equivalently, the following equation

$$
u(t)+\int_{0}^{t}(\nu \widehat{A} u(s)+\widehat{B}(u(s), u(s))) d s=u_{0}+\int_{0}^{t} \widehat{F}(u(s), s) d s+\int_{0}^{t} \widehat{G}(u(s), s) d W(s),
$$

holds in $\mathbb{W}^{\star} P$-a.s. for any $t \in(0, T)$.

Remark 3.3. In the above definition the quantity $\int_{0}^{t}(G(u(s), s), v) d W(s)$ should be understood as

$$
\int_{0}^{t}(G(u(s), s), v) d W(s)=\sum_{k=1}^{m} \int_{0}^{t}\left(G_{k}(u(s), s), v\right) d W_{k}(s),
$$

where $G_{k}$ and $W_{k}$ denote the $k$-th component of $G$ and $W$, respectively.

Now we are ready to formulate the main result of this section.

Theorem 3.4. Assume that $u_{0} \in \mathbb{W}$ is non-random and that all the assumptions, namely (17)- (20), on the operators $F$ and $G$ are satisfied, then the problem (1) has a solution in the sense of the above definition. Moreover, almost surely the stochastic process u has $\mathbb{W}($ resp., $\mathbb{V})$-valued weak(resp., strong) continuous paths.

Remark 3.5. The theorem still holds if we assume that $u_{0}$ is $\mathcal{F}^{0}$-measurable and satisfies $E\left|u_{0}\right|_{\mathbb{W}}^{p}<\infty, 2 \leq p<\infty$. 


\subsection{Proof of Theorem 3.4}

This subsection is devoted to the proof of the existence result stated in the preceding subsection. We split the proof into two parts.

\section{Part 1: The Approximate solution and some a priori estimates}

In this part we introduce the Galerkin approximations of our problem and give crucial estimates which will serve as a toolkit for the proof of the Theorem 3.4. We first note that the injection of $\mathbb{W}$ into $\mathbb{V}$ is compact. Let $I$ be the isomorphism of $\mathbb{W}{ }^{\star}$ onto $\mathbb{W}$, then the restriction of $I$ to $\mathbb{V}$ is a continuous compact operator into itself. Thus, There exists a sequence $\left\{e_{i}: i=1,2,3, \ldots\right\}$ of elements of $\mathbb{W}$ which forms an orthonormal basis in $\mathbb{W}$, and an orthogonal basis in $\mathbb{V}$. The elements of this sequence are the solutions of the eigenvalue problem

$$
\left(v, e_{i}\right)_{\mathbb{W}}=\lambda_{i}\left(v, e_{i}\right)_{\mathbb{V}}, \text { for any } v \in \mathbb{W} .
$$

Here $\left\{\lambda_{i}: i=1,2,3, \ldots\right\}$ is an increasing sequence of positive eigenvalues. We have the important result due to [16] about the regularity of the functions $e_{i}, i=1,2,3, \ldots$.

Lemma 3.6. Let $D$ be a bounded, simply-connected open set of $\mathbb{R}^{2}$ with a boundary of class $\mathcal{C}^{3}$, then the eigenfunctions of (24) belong to $\mathbb{H}^{4} \mathcal{O}$.

We now introduce the Galerkin approximation scheme for the problem (1). We consider the subset $\mathbb{W}_{N}=\operatorname{Span}\left(e_{1}, \ldots, e_{N}\right) \subset \mathbb{W}$ and look for a finite-dimensional approximation of a solution of our problem as a vector $u^{N} \in \mathbb{W}_{N}$ that can be written as a Fourier series:

$$
u^{N}(t)=\sum_{i=1}^{N} c_{i N}(t) e_{i}(x) .
$$

We require $u^{N}$ to satisfy the following system

$$
\begin{aligned}
& d\left(u^{N}, e_{i}\right)_{\mathbb{V}}+\nu\left(\left(u^{N}, e_{i}\right)\right) d t+b\left(u^{N}, u^{N}, e_{i}\right) d t-\alpha b\left(u^{N}, \Delta u^{N}, e_{i}\right) d t+\alpha b\left(e_{i}, \Delta u^{N}, u^{N}\right) d t \\
& =\left(F\left(u^{N}, t\right), e_{i}\right) d t+\left(G\left(u^{N}, t\right), e_{i}\right) d W, i \in\{1, \ldots, N\}
\end{aligned}
$$

or equivalently

$$
d\left(u^{N}, e_{i}\right)_{\mathbb{V}}+\nu\left(\widehat{A} u^{N}, e_{i}\right)_{\mathbb{V}} d t+\left\langle\widehat{B}\left(u^{N}, u^{N}\right), e_{i}\right\rangle d t=\left(\widehat{F}\left(u^{N}, t\right), e_{i}\right)_{\mathbb{V}} d t+\left(\widehat{G}\left(u^{N}, t\right), e_{i}\right)_{\mathbb{V}} d W
$$

for any $i \in\{1, . ., N\}$. The function $u_{0}^{N}$ is the orthogonal projection of $u_{0}$ onto the space $\mathbb{W}_{N}$,

$$
u_{0}^{N} \rightarrow u_{0} \text { strongly in } \mathbb{V} \text { as } N \rightarrow \infty .
$$

The Fourier coefficients $c_{i N}$ in (25) are solutions of a system of stochastic ordinary differential equations with locally Lipschitz coefficients. By well known existence and uniqueness theorem on system of stochastic differential equations (see for example [49],[32]...), a sequence of continuous functions $u^{N}$ exists at least on a short interval $\left(0, T_{N}\right)$. Global existence will follow from a priori estimates for $u^{N}$ (see Lemma 3.7).

From now on, we denote by $C$ any constant depending only on the data, and which may change from one line to the next one. The following result can be proved exactly as in [41] (see proof of Lemma 4.2 and Lemma 4.3 in [41]).

Lemma 3.7. The sequence $u^{N}$ satisfies

$$
E \sup _{0 \leq s \leq T}\left|u^{N}(s)\right|_{\mathbb{W}}^{p}<C, \text { for any } 2 \leq p<\infty .
$$




\section{Part 2: Passage to the limits and proof of the continuity}

To complete the proof of the existence of solution we need to pass to the limit in the Galerkin approximation.

Owing to (28) we can extract by a diagonal argument a subsequence $u^{N_{\mu}}$ such that

$$
u^{N_{\mu}} \rightarrow u \text { weakly-* in } L^{p}\left(\Omega, \mathcal{F}, P, L^{\infty}(0, T, \mathbb{W})\right),
$$

for any $2 \leq p<\infty$ as $N_{\mu} \rightarrow \infty$. It also follows from (28) that as $N_{\mu} \rightarrow \infty$

$$
u^{N_{\mu}} \rightarrow u \text { weakly in } L^{p}\left(\Omega, \mathcal{F}, P, L^{q}(0, T, \mathbb{V})\right),
$$

for any $2 \leq p, q<\infty$ as $N_{\mu} \rightarrow \infty$. Thanks to (13), (17)-(20) all together with (28) there exist three operators $\widehat{B}^{*}(s), F^{*}(s)$ and $G^{*}(s)$ such that

$$
\begin{aligned}
A u^{N_{\mu}} & \rightarrow A u \text { weakly in } L^{2}\left(\Omega, \mathcal{F}, P, L^{2}(0, T, \mathbb{V})\right), \\
\widehat{B}\left(u^{N_{\mu}}, u^{N_{\mu}}\right) & \rightarrow \widehat{B}^{*}(s) \text { weakly in } L^{2}\left(\Omega, \mathcal{F}, P, L^{2}\left(0, T, \mathbb{W}^{*}\right)\right), \\
F\left(u^{N_{\mu}}, s\right) & \rightarrow F^{*}(s) \text { weakly in } L^{2}\left(\Omega, \mathcal{F}, P, L^{2}(0, T, \mathbb{V})\right), \\
G\left(u^{N_{\mu}}, s\right) & \rightarrow G^{*}(s) \text { weakly in } L^{2}\left(\Omega, \mathcal{F}, P, L^{2}\left(0, T, \mathbb{V}^{\otimes m}\right)\right) .
\end{aligned}
$$

It follows from the properties of the linear operator $(I+\alpha A)^{-1}$ that

$$
\begin{gathered}
\widehat{A} u^{N_{\mu}} \rightarrow \widehat{A} u \text { weakly in } L^{2}\left(\Omega, \mathcal{F}, P, L^{2}(0, T, \mathbb{V})\right) \\
\widehat{F}\left(u^{N_{\mu}}, s\right) \rightarrow \widehat{F}^{*}(s) \text { weakly in } L^{2}\left(\Omega, \mathcal{F}, P, L^{2}(0, T, \mathbb{V})\right), \\
\widehat{G}\left(u^{N_{\mu}}, s\right) \rightarrow \widehat{G}^{*}(s) \text { weakly in } L^{2}\left(\Omega, \mathcal{F}, P, L^{2}\left(0, T, \mathbb{V}^{\otimes m}\right)\right),
\end{gathered}
$$

where $\widehat{F}^{*}\left(\right.$ resp. $\left.\widehat{G}^{*}\right)$ denotes $(I+\alpha A)^{-1} F^{*}\left(\right.$ resp. $\left.(I+\alpha A)^{-1} G^{*}\right)$. With these convergences at hand we see from (27) that the following holds

$$
\begin{array}{r}
\left(u(t), e_{i}\right)_{\mathbb{V}}+\int_{0}^{t}\left(\nu\left(\widehat{A} u(s), e_{i}\right)_{\mathbb{V}}+\left\langle\widehat{B}^{*}(s), e_{i}\right\rangle\right) d s=\left(u_{0}, e_{i}\right)_{\mathbb{V}}+\int_{0}^{t}\left(\widehat{F}^{*}(s), e_{i}\right)_{\mathbb{V}} d s \\
+\int_{0}^{t}\left(\widehat{G}^{*}(s), e_{i}\right)_{\mathbb{V}} d W(s),
\end{array}
$$

for any $t \in[0, T], i \in\{1, . ., N\}$, and for almost all $\omega \in \Omega$.

Arguing as in [38] (see Chapter 2, Page 42) we deduce from the latter equation that almost all paths of $u$ are $\mathbb{V}$-valued continuous functions. From (29) we see that

$$
u \in L^{2}\left(\Omega, \mathcal{F}, P ; L^{\infty}(0, T ; \mathbb{V})\right) .
$$

Therefore we can use the argument in [52](Chap. 3, Section 1) to assert that almost all paths of $u$ are weakly continuous as functions taking values in $\mathbb{W}$. To complete the proof of Theorem 3.4 we need the following result.

Lemma 3.8. We have the following identities

$$
\begin{aligned}
& \widehat{B}^{*}(s)=\widehat{B}(u, u) \text { in } L^{2}\left(\Omega, \mathcal{F}, P, L^{2}\left(0, T, \mathbb{W}^{*}\right)\right), \\
& \widehat{F}^{*}(s)=\widehat{F}(u, s) \text { in } L^{2}\left(\Omega, \mathcal{F}, P, L^{2}(0, T, \mathbb{V})\right), \\
& \widehat{G}^{*}(s)=\widehat{G}(u, s) \text { in } L^{2}\left(\Omega, \mathcal{F}, P, L^{2}\left(0, T, \mathbb{V}^{\otimes m}\right)\right)
\end{aligned}
$$


For $M \geq 1$ let us define the sequence of stopping times $\left\{\tau_{M} ; M \geq 1\right\}$ by

$$
\inf \left\{t ;|u(t)|_{\mathbb{V}}+|u(t)|_{*} \geq M\right\} \wedge T,
$$

where $|u(t)|_{*}=|\operatorname{curl}(u(t)-\alpha \Delta u(t))|$.

The proof of Lemma 3.8 require the following results.

Lemma 3.9. For any $M \geq 1$ we have that

$$
1_{\left[0, \tau_{M}\right]}\left(u^{N}-u\right) \rightarrow 0 \text { strongly in } L^{2}\left(\Omega, \mathcal{F}, P ; L^{2}(0, T ; \mathbb{V})\right),
$$

as $N \rightarrow \infty$.

Proof. We follow some arguments used in [8] and [14] (see also [22])). From now on we set $N:=N_{\mu}, \widehat{F}(x, t):=\widehat{F}(x)$, and $\widehat{G}(x, t):=\widehat{G}(x)$ for any $x \in \mathbb{V}$ and $t \in[0, T]$. Let $P^{N}: \mathbb{W} \rightarrow \mathbb{W}_{N}$ be the orthogonal projection defined by

$$
P^{N} v=\sum_{i=1}^{N}\left(v, e_{i}\right)_{\mathbb{W}} e_{i}=\sum_{i=0}^{N} \lambda_{i}\left(v, e_{i}\right)_{\mathbb{V}} e_{i}, v \in \mathbb{W} .
$$

For any element $v$ in $L^{2}\left(\Omega, \mathcal{F}, P ; L^{2}(0, T ; \mathbb{W})\right)$, there holds

$$
\begin{array}{r}
\left|P^{N} v-v\right|_{\mathbb{W}}=\left|\sum_{i=1}^{N}\left(v, e_{i}\right) \mathbb{W} e_{i}-\sum_{i=1}^{\infty}\left(v, e_{i}\right)_{\mathbb{W}} e_{i}\right|_{\mathbb{W}} \\
\leq\left|\sum_{i=N+1}^{\infty}\left(v, e_{i}\right)_{\mathbb{W}} e_{i}\right|_{\mathbb{W}},
\end{array}
$$

for almost all $(\omega, t)$ in $\Omega \times(0, T)$. Since the family $\left\{e_{i}: i=1,2,3, \ldots\right\}$ forms an orthonormal basis of $\mathbb{W}$ (see the construction of the the special basis in the Part 1 of the proof), then

$$
P^{N} v(\omega, t) \rightarrow v(\omega, t) \text { strongly in } \mathbb{W} \text { for almost all }(\omega, t) \in \Omega \times(0, T) .
$$

We also have

$$
\left|P^{N} v\right|_{\mathbb{W}}^{2} \leq C|v|_{\mathbb{W}}^{2}
$$

Therefore, by the Dominated Convergence Theorem we see that

$$
P^{N} v \rightarrow v \text { strongly in } L^{2}\left(\Omega, \mathcal{F}, P ; L^{2}(0, T ; \mathbb{W})\right) .
$$

Thanks to the continuous embedding $L^{2}\left(\Omega, \mathcal{F}, P ; L^{2}(0, T ; \mathbb{W})\right) \hookrightarrow L^{2}\left(\Omega, \mathcal{F}, P ; L^{2}(0, T ; \mathbb{V})\right)$, we also have

$$
P^{N} v \rightarrow v \text { strongly in } L^{2}\left(\Omega, \mathcal{F}, P ; L^{2}(0, T ; \mathbb{V})\right) .
$$

For any $v \in \mathbb{W}$, there holds

$$
\begin{aligned}
\left(P^{N} v, e_{i}\right) & =\left(\sum_{i=1}^{N}\left(v, e_{j}\right)_{\mathbb{W}} e_{j}, e_{i}\right)_{\mathbb{V}}, \\
& =\left(v, e_{i}\right)_{\mathbb{W}}\left(e_{i}, e_{i}\right)_{\mathbb{V}},
\end{aligned}
$$

where we have used the fact that $\left\{e_{i}: i=1,2,3, \ldots\right\}$ forms an orthogonal basis of $\mathbb{V}$. From the property (24)

$$
\left(P^{N} v, e_{i}\right)_{\mathbb{V}}=\left(v, e_{i}\right)_{\mathbb{V}} \lambda_{i}\left(e_{i}, e_{i}\right)_{\mathbb{V}}
$$


Using again (24), we find that

$$
\left(P^{N} v, e_{i}\right)_{\mathbb{V}}=\left(v, e_{i}\right)_{\mathbb{V}}
$$

for any $v \in \mathbb{W}$. Out of this and (33), we deduce that

$$
\begin{array}{r}
\left(P^{N} u(t), e_{i}\right)_{\mathbb{V}}+\int_{0}^{t}(\nu \\
\left.=\int_{0}^{N} \widehat{A} u(s), e_{i}\right)_{\mathbb{V}}+\left\langle\widehat{B}^{*}(s), e_{i}\right)_{\mathbb{V}} d s+\int_{0}^{t}\left(\widehat{G}^{*}(s), e_{i}\right)_{\mathbb{V}} d W(s),
\end{array}
$$

for any $t \in[0, T], i \in\{1, . ., N\}$. This estimate and (27) imply that

$$
\begin{aligned}
& \left(P^{N} u(t)-u^{N}(t), e_{i}\right)_{\mathbb{V}}+\int_{0}^{t} \nu\left(P^{N} \widehat{A}\left(u(s)-u^{N}(s)\right), e_{i}\right)_{\mathbb{V}} \\
& =\int_{0}^{t}\left\langle\widehat{B}\left(u^{N}(s), u^{N}(s)\right)-\widehat{B}^{*}(s), e_{i}\right\rangle d s+\int_{0}^{t}\left(\widehat{F}^{*}(s)-\widehat{F}\left(u^{N}\right), e_{i}\right)_{\mathbb{V}} d s \\
& +\int_{0}^{t}\left(\widehat{G}^{*}(s)-\widehat{G}\left(u^{N}\right), e_{i}\right)_{\mathbb{V}} d W(s) .
\end{aligned}
$$

We apply Itô's formula to the function $\left(P^{N} u(t)-u^{N}(t), e_{i}\right)_{\mathbb{V}}^{2}$, then we multiply the resulting equation by $\lambda_{i}$ and sum over $i$ from 1 to $N$ to obtain

$$
\begin{aligned}
& \left|P^{N} u-u^{N}\right|_{\mathbb{V}}^{2}+2 \nu \int_{0}^{t}\left(P^{N} \widehat{A}\left(u-u^{N}\right), P^{N} u-u^{N}\right)_{\mathbb{V}} \\
& =2 \int_{0}^{t}\left\langle\widehat{B}\left(u^{N}, u^{N}\right)-\widehat{B}^{*}(s), P^{N} u-u^{N}\right\rangle d s+2 \int_{0}^{t}\left(\widehat{F}^{*}(s)-\widehat{F}\left(u^{N}\right), P^{N} u-u^{N}\right)_{\mathbb{V}} d s \\
& +\sum_{i=1}^{N} \lambda_{i} \int_{0}^{t}\left(\widehat{G}^{*}(s)-\widehat{G}\left(u^{N}\right), e_{i}\right)_{\mathbb{V}}^{2} d s+2 \int_{0}^{t}\left(\widehat{G}^{*}(s)-\widehat{G}\left(u^{N}\right), P^{N} u-u^{N}\right)_{\mathbb{V}} d W(s) .
\end{aligned}
$$

Here we have set $u(s)=u, u^{N}(s)=u^{N} ; s \in[0, T]$. Now let

$$
\sigma(t)=\exp \left(-\eta_{1} t-\eta_{2} \int_{0}^{t}|u(s)|_{\mathbb{W}}^{2} d s\right)
$$

where $\eta_{1}$ and $\eta_{2}$ are two positive constants to be defined later. By applying Itô's formula to the process $\sigma(t)\left|P^{N} u-u^{N}\right|_{\mathbb{V}}^{2}$ we arrive at

$$
\begin{aligned}
& \sigma(t)\left|P^{N} u-u^{N}\right|_{\mathbb{V}}^{2}+2 \nu \int_{0}^{t} \sigma(s)\left(\widehat{A}\left(u-u^{N}\right), P^{N} u-u^{N}\right)_{\mathbb{V}} \\
= & \sum_{i=1}^{N} \lambda_{i} \int_{0}^{t} \sigma(s)\left(\widehat{G}^{*}(s)-\widehat{G}\left(u^{N}\right), e_{i}\right)_{\mathbb{V}}^{2} d s+2 \int_{0}^{t} \sigma(s)\left(\widehat{G}^{*}(s)-\widehat{G}\left(u^{N}\right), P^{N} u-u^{N}\right)_{\mathbb{V}} d W(s) \\
& -\eta_{1} \int_{0}^{t} \sigma(s)\left|P^{N} u-u^{N}\right|_{\mathbb{V}}^{2} d s-\eta_{2} \int_{0}^{t} \sigma(s)\left|P^{N} u-u^{N}\right|_{\mathbb{V}}^{2}|u(s)|_{\mathbb{W}}^{2} d s \\
& +2 \int_{0}^{t} \sigma(s)\left\langle\widehat{B}\left(u^{N}, u^{N}\right)-\widehat{B}^{*}(s), P^{N} u-u^{N}\right\rangle d s \\
& +2 \int_{0}^{t} \sigma(s)\left(\widehat{F}^{*}(s)-\widehat{F}\left(u^{N}\right), P^{N} u-u^{N}\right)_{\mathbb{V}} d s .
\end{aligned}
$$


Let us analyze each term of this equation. First, we have that

$$
\left(\widehat{A}\left(u-u^{N}\right), P^{N} u-u^{N}\right)_{\mathbb{V}}=\left(\widehat{A}\left(u-u^{N}\right), u-u^{N}\right)_{\mathbb{V}}-\left(\widehat{A}\left(u-u^{N}\right), u-P^{N} u\right)_{\mathbb{V}} .
$$

By the definition of $P^{N}$ we have

$$
P^{N} u^{N}=u^{N}
$$

and

$$
\left|P^{N}\left(u-u^{N}\right)\right|_{\mathbb{V}} \leq\left|u-u^{N}\right|_{\mathbb{V}}
$$

Then we deduce from (8), (3) together with these properties of $P^{N}$ that

$$
\frac{1}{\mathcal{P}^{2}+\alpha}\left|P^{N} u-u^{N}\right|_{\mathbb{V}}^{2} \leq\left(\widehat{A}\left(u-u^{N}\right), u-u^{N}\right)_{\mathbb{V}}
$$

Second, by (21) the following holds:

$$
\begin{aligned}
2\left(\widehat{F}^{*}(s)-\widehat{F}\left(u^{N}\right), P^{N} u-u^{N}\right)_{\mathbb{V}} \leq \frac{4 \mathcal{P}^{2} C_{F}}{\alpha}\left|u^{N}-P^{N} u\right|_{\mathbb{V}}^{2} & +\frac{2 \mathcal{P}^{2} C_{F}}{\alpha}\left|P^{N} u-u\right|_{\mathbb{V}}^{2} \\
& +2\left(\widehat{F}^{*}(s)-\widehat{F}(u), P^{N} u-u^{N}\right)_{\mathbb{V} .}
\end{aligned}
$$

Third, let us set

$$
\mathcal{G}=\sum_{i=0}^{N} \lambda_{i}\left(\widehat{G}\left(u^{N}\right)-\widehat{G}^{*}(s), e_{i}\right)_{\mathbb{V}}^{2}
$$

We have that

$$
\mathcal{G}=\left|P^{N}\left[\widehat{G}\left(u^{N}\right)-\widehat{G}^{*}(s)\right]\right|_{\mathbb{V}}^{2},
$$

from which we derive that

$$
\begin{array}{r}
\mathcal{G}=\left|P^{N}\left[\widehat{G}(u)-\widehat{G}\left(u^{N}\right)\right]\right|_{\mathbb{V} \otimes m}^{2}-\left|P^{N}\left[\widehat{G}(u)-\widehat{G}^{*}(s)\right]\right|_{\mathbb{V} \otimes m}^{2} \\
+2\left(P^{N}\left[\widehat{G}^{*}(s)-\widehat{G}\left(u^{N}\right)\right], P^{N}\left[\widehat{G}^{*}(s)-\widehat{G}(u)\right]\right)_{\mathbb{V}} .
\end{array}
$$

It follows from (22) that

$$
\begin{aligned}
\mathcal{G} \leq 2 C_{G}\left|P^{N} u-u\right|_{\mathbb{V}}^{2} & +2 C_{G}\left|P^{N} u-u^{N}\right|_{\mathbb{V}}^{2}-\left|P^{N}\left[\widehat{G}(u)-\widehat{G}^{*}(s)\right]\right|_{\mathbb{V} \otimes m}^{2} \\
& +2\left(P^{N}\left[\widehat{G}^{*}(s)-\widehat{G}\left(u^{N}\right)\right], P^{N}\left[\widehat{G}^{*}(s)-\widehat{G}(u)\right]\right)_{\mathbb{V}} .
\end{aligned}
$$

Fourth, we can check that the following holds

$$
\begin{array}{r}
\left\langle\widehat{B}\left(u^{N}, u^{N}\right), P^{N} u-u^{N}\right\rangle=\left\langle\widehat{B}\left(P^{N} u-u^{N}, P^{N} u-u^{N}\right), P^{N} u\right\rangle \\
+\left\langle\widehat{B}\left(P^{N} u, P^{N} u\right), P^{N} u-u^{N}\right\rangle .
\end{array}
$$

Indeed, the property (15) implies that

$$
\begin{array}{r}
\left\langle\widehat{B}\left(P^{N} u-u^{N}, P^{N} u-u^{N}\right), P^{N} u\right\rangle=\left\langle\widehat{B}\left(u^{N}, u^{N}\right), P^{N} u-u^{N}\right\rangle \\
\\
-\left\langle\widehat{B}\left(P^{N} u, u^{N}\right), P^{N} u\right\rangle,
\end{array}
$$

and

$$
-\left\langle\widehat{B}\left(P^{N} u, u^{N}\right), P^{N} u\right\rangle=\left\langle\widehat{B}\left(P^{N} u, P^{N} u-u^{N}\right), P^{N} u\right\rangle .
$$

Taking (49) into (48) and the application of (16) yield (47). We deduce from (47) along with the property (11) that

$$
\begin{aligned}
2\left\langle\widehat{B}\left(u^{N}, u^{N}\right), P^{N} u-u^{N}\right\rangle \leq & 2 C_{B}\left|P^{N} u-u^{N}\right|_{\mathbb{V}}^{2}\left|P^{N} u\right|_{\mathbb{W}} \\
& +2\left\langle\widehat{B}\left(P^{N} u, P^{N} u\right), P^{N} u-u^{N}\right\rangle .
\end{aligned}
$$


Therefore

$$
\begin{aligned}
2\left\langle\widehat{B}\left(u^{N}, u^{N}\right)-\widehat{B}^{*}(s), P^{N} u-u^{N}\right\rangle \leq & C_{B}^{2}\left|P^{N} u-u^{N}\right|_{\mathbb{V}}^{2}\left|P^{N} u\right|_{\mathbb{W}}^{2}+\left|u^{N}-P^{N} u\right|_{\mathbb{V}}^{2} \\
& +2\left\langle\widehat{B}\left(P^{N} u, P^{N} u\right)-\widehat{B}^{*}(s), P^{N} u-u^{N}\right\rangle .
\end{aligned}
$$

Using (43) and (44) in (42) one gets

$$
\begin{aligned}
& \sigma(t)\left|P^{N} u(t)-u^{N}(t)\right|_{\mathbb{V}}^{2}+\frac{2 \nu}{\mathcal{P}^{2}+\alpha} \int_{0}^{t} \sigma(s)\left|P^{N} u-u^{N}\right|_{\mathbb{V}}^{2} d s \\
& \leq R H S(t)+2 \nu \int_{0}^{t} \sigma(s)\left(\widehat{A}\left(u-u^{N}\right), u-P^{N} u\right)_{\mathbb{V}} d s,
\end{aligned}
$$

where $R H S(t)$ denotes the right hand side of (42) . Now, by choosing

$$
\begin{aligned}
\eta_{1} & =\frac{4 \mathcal{P}^{2} C_{F}}{\alpha}+2 C_{G}+1, \\
\eta_{2} & =C_{B}^{2},
\end{aligned}
$$

we can infer from (51), (50), (46) and (45) that

$$
\begin{aligned}
& \sigma(t)\left|P^{N} u(t)-u^{N}(t)\right|_{\mathbb{V}}^{2}+\int_{0}^{t} \sigma(s)\left(\frac{2 \nu}{\mathcal{P}^{2}+\alpha}\left|P^{N} u-u^{N}\right|_{\mathbb{V}}^{2}+\left|P^{N}\left[\widehat{G}(u)-\widehat{G}^{*}(s)\right]\right|_{\mathbb{V} \otimes m}^{2}\right) d s \\
& \leq 2 \nu \int_{0}^{t} \sigma(s)\left(\widehat{A}\left(u-u^{n}\right), u-P^{N} u\right)_{\mathbb{V}} d s+C \int_{0}^{t} \sigma(s)\left|P^{N} u-u\right|_{\mathbb{V}}^{2} d s \\
& \quad+2 \int_{0}^{t} \sigma(s)\left\langle\widehat{B}\left(P^{N} u, P^{N} u\right)-\widehat{B}^{*}(s), P^{N} u-u^{N}\right\rangle d s \\
& \quad+2 \int_{0}^{t} \sigma(s)\left(\widehat{F}^{*}(s)-\widehat{F}(u), P^{N} u-u^{N}\right)_{\mathbb{V}} d s \\
& \quad+2 \int_{0}^{t} \sigma(s)\left(P^{N}\left[\widehat{G}^{*}(s)-\widehat{G}\left(u^{N}\right)\right], P^{N}\left[\widehat{G}^{*}(s)-\widehat{G}(u)\right]\right)_{\mathbb{V}} d s \\
& \quad+2 \int_{0}^{t} \sigma(s)\left(\widehat{G}^{*}(s)-\widehat{G}\left(u^{N}\right), P^{N} u-u^{N}\right)_{\mathbb{V}} d W .
\end{aligned}
$$

Replacing $t$ by $\tau_{M}$ in the estimate (52), and taking the mathematical expectation yield

$$
\begin{aligned}
& E \sigma\left(\tau_{M}\right)\left|P^{N} u\left(\tau_{M}\right)-u^{N}\left(\tau_{M}\right)\right|_{\mathbb{V}}^{2}+C E \int_{0}^{\tau_{M}} \sigma(s)\left(\left|P^{N} u-u^{N}\right|_{\mathbb{V}}^{2}-\left|P^{N} u-u\right|_{\mathbb{V}}^{2}\right) d s \\
\leq & -E \int_{0}^{\tau_{M}} \sigma(s)\left|P^{N}\left[\widehat{G}(u)-\widehat{G}^{*}(s)\right]\right|_{\mathbb{V} \otimes m}^{2} d s+2 \nu E \int_{0}^{\tau_{M}} \sigma(s)\left(\widehat{A}\left(u-u^{n}\right), u-P^{N} u\right)_{\mathbb{V}} d s \\
& +2 E \int_{0}^{\tau_{M}} \sigma(s)\left(P^{N}\left[\widehat{G}^{*}(s)-\widehat{G}\left(u^{N}\right)\right], P^{N}\left[\widehat{G}^{*}(s)-\widehat{G}(u)\right]\right)_{\mathbb{V}} d s \\
& +2 E \int_{0}^{\tau_{M}} \sigma(s)\left\langle\widehat{B}\left(P^{N} u, P^{N} u\right)-\widehat{B}^{*}(s), P^{N} u-u^{N}\right\rangle d s \\
& +2 E \int_{0}^{\tau_{M}} \sigma(s)\left(\widehat{F}^{*}(s)-\widehat{F}(u), P^{N} u-u^{N}\right)_{\mathbb{V}} d s .
\end{aligned}
$$

Since

$$
\begin{aligned}
\left|1_{\left[0, \tau_{M}\right]} \sigma(t)\left[\widehat{B}\left(P^{N} u(t), P^{N} u(t)\right)-\widehat{B}(u(t), u(t))\right]\right|_{\mathbb{W}^{*}} \leq 1_{\left[0, \tau_{M}\right]} C_{B}|u(t)|_{\mathbb{W}}\left|P^{N} u(t)-u(t)\right|_{\mathbb{W}} \\
+1_{\left[0, \tau_{M}\right]} C_{B}|u(t)|_{\mathbb{W}}\left|P^{N} u(t)-u(t)\right|_{\mathbb{V}}
\end{aligned}
$$


then

$$
\left|1_{\left[0, \tau_{M}\right]} \sigma(t)\left[\widehat{B}\left(P^{N} u(t), P^{N} u(t)\right)-\widehat{B}(u(t), u(t))\right]\right|_{\mathbb{W}^{*}} \rightarrow 0 \text { a.e. }(\omega, t) \in \Omega \times[0, T],
$$

as $N \rightarrow \infty$. Furthermore

$$
\left|1_{\left[0, \tau_{M}\right]} \sigma(t)\left[\widehat{B}\left(P^{N} u(t), P^{N} u(t)\right)-\widehat{B}(u(t), u(t))\right]\right|_{\mathbb{W} *} \leq 2 M C_{B} C^{*}|u(t)|_{\mathbb{W}}^{2},
$$

where $C^{*}$ is the constant in the continuous embedding $\mathbb{W} \hookrightarrow \mathbb{V}$. Note that $|u(t)|_{\mathbb{W}}^{2}$ is bounded in $\in L^{2}(\Omega \times[0, T], \mathbb{R})$. By the convergences (29), (39) we have

$$
P^{N} u-u^{N} \rightarrow 0 \text { weakly in } L^{2}\left(\Omega, \mathcal{F}, P ; L^{2}(0, T ; \mathbb{W})\right) .
$$

We derive from this, (53) and (54) that

$$
E \int_{0}^{\tau_{M}} \sigma(s)\left\langle\widehat{B}\left(P^{N} u, P^{N} u\right)-\widehat{B}(u, u), P^{N} u-u^{N}\right\rangle d s \rightarrow 0
$$

as $N \rightarrow \infty$. Hence

$$
\begin{aligned}
& \lim _{N \rightarrow \infty} E \int_{0}^{\tau_{M}} \sigma(s)\left\langle\widehat{B}\left(P^{N} u, P^{N} u\right)-\widehat{B}^{*}(s), P^{N} u-u^{N}\right\rangle d s \\
= & \lim _{N \rightarrow \infty} E \int_{0}^{\tau_{M}} \sigma(s)\left\langle\widehat{B}\left(P^{N} u, P^{N} u\right)-\widehat{B}(u, u), P^{N} u-u^{N}\right\rangle d s \\
& +\lim _{N \rightarrow \infty} E \int_{0}^{\tau_{M}} \sigma(s)\left\langle\widehat{B}(u, u)-\widehat{B}^{*}(s), P^{N} u-u^{N}\right\rangle d s \\
= & 0
\end{aligned}
$$

Owing to (31) the sequence $\widehat{A}\left(u-u^{N}\right)$ is bounded in $L^{2}\left(\Omega, \mathcal{F}, P ; L^{2}(0, T ; \mathbb{V})\right)$, thus

$$
E \int_{0}^{\tau_{M}} \sigma(s)\left(\widehat{A}\left(u-u^{N}\right), P^{N} u-u\right)_{\mathbb{V}} d s \rightarrow 0 \text { as } N \rightarrow \infty .
$$

We also show that

$$
E \int_{0}^{\tau_{M}} \sigma(s)\left(\widehat{F}^{*}(s)-\widehat{F}(u), P^{N} u-u^{N}\right)_{\mathbb{V}} d s \rightarrow 0 \text { as } N \rightarrow \infty .
$$

The convergence (40) implies that

$$
\lim _{N \rightarrow 0} E \int_{0}^{\tau_{M}} \sigma(s)\left|P^{N} u-u\right|_{\mathbb{V}}^{2} d s=0 .
$$

Since

$$
1_{\left[0, \tau_{M}\right]} \sigma(s) \widehat{G}^{*}(s)-\widehat{G}(u) \in L^{2}\left(\Omega, \mathcal{F}, P ; L^{2}(0, T ; \mathbb{V})\right),
$$

then we derive from (32) that

$$
\lim _{n \rightarrow \infty} E \int_{0}^{\tau_{M}} \sigma(s)\left(P^{N}\left[\widehat{G}^{*}(s)-\widehat{G}\left(u^{N}\right)\right], P^{N}\left[\widehat{G}^{*}(s)-\widehat{G}(u)\right]\right)_{\mathbb{V}} d s=0 .
$$

The convergences (55)-(58) imply that

$$
\begin{aligned}
& E \sigma\left(\tau_{M}\right)\left|P^{N} u\left(\tau_{M}\right)-u^{N}\left(\tau_{M}\right)\right|_{\mathbb{V}}^{2}+C E \int_{0}^{\tau_{M}} \sigma(s)\left|P^{N} u-u^{N}\right|_{\mathbb{V}}^{2} d s+ \\
& +E \int_{0}^{\tau_{M}} \sigma(s)\left|P^{N}\left[\widehat{G}(u)-\widehat{G}^{*}(s)\right]\right|_{\mathbb{V} \otimes m}^{2} \rightarrow 0
\end{aligned}
$$

as $N \rightarrow \infty$. The proof of the lemma follows from (40) and from (59). 
Proof of Lemma 3.8. Now we complete the proof of the identities (34)-(36). First note that for any $w \in \mathbb{W}$

$$
\begin{aligned}
S & =\left\langle\widehat{B}\left(u^{N}, u^{N}\right)-\widehat{B}(u, u), w\right\rangle \\
& =\left\langle\widehat{B}\left(u^{N}-u, u^{N}\right), w\right\rangle+\left\langle\widehat{B}\left(u, u^{N}-u\right), w\right\rangle .
\end{aligned}
$$

The following equations also hold true

$$
\begin{aligned}
& \left\langle\widehat{B}\left(u^{N}-u, u^{N}\right), w\right\rangle=\left\langle\widehat{B}\left(u^{N}, u^{N}\right), w\right\rangle-\left\langle\widehat{B}\left(u, u^{N}\right), w\right\rangle, \\
& \left\langle\widehat{B}\left(u^{N}, u-u^{N}\right), w\right\rangle=\left\langle\widehat{B}\left(u^{N}, u\right), w\right\rangle-\left\langle\widehat{B}\left(u^{N}, u^{N}\right), w\right\rangle .
\end{aligned}
$$

Therefore

$$
\begin{aligned}
S=\left\langle\widehat{B}\left(u, u-u^{N}\right), w\right\rangle-\left\langle\widehat{B}\left(u^{N}, u-u^{N}\right), w\right\rangle & +\left\langle\widehat{B}\left(u^{N}, u\right), w\right\rangle \\
& -\left\langle\widehat{B}\left(u, u^{N}\right), w\right\rangle .
\end{aligned}
$$

Thanks to (11) the operator

$$
\begin{aligned}
\widehat{B}_{a, .}: \mathbb{V} & \rightarrow \mathbb{W}^{*} \\
v & \mapsto \widehat{B}_{a, .}(v)=\widehat{B}(a, v)
\end{aligned}
$$

is linear continuous for any fixed $a \in \mathbb{W}$. Due to this fact and (30), it is true that

$$
\widehat{B}\left(u, u^{N}\right) \rightarrow \widehat{B}(u, u) \text { weakly in } L^{2}\left(\Omega, \mathcal{F}, P ; L^{2}\left(0, T ; \mathbb{W}^{*}\right)\right) .
$$

By a similar argument, we also prove the following convergence

$$
\widehat{B}\left(u^{N}, u\right) \rightarrow \widehat{B}(u, u) \text { weakly in } L^{2}\left(\Omega, \mathcal{F}, P ; L^{2}\left(0, T ; \mathbb{W}^{*}\right)\right) .
$$

Now let $w$ be an element of $L^{\infty}\left(\Omega, \mathcal{F}, P ; L^{\infty}(0, T ; \mathbb{W})\right)$. We deduce from the property (13) that

$$
\begin{aligned}
& \left|E \int_{0}^{T} 1_{\left[0, \tau_{M}\right]}\left\langle\widehat{B}\left(u, u-u^{N}\right), w(s)\right\rangle-\left\langle\widehat{B}\left(u^{N}, u-u^{N}\right), w(s)\right\rangle d s\right| \\
& \leq C E \int_{0}^{\tau_{M}}|u|_{\mathbb{W}}\left|u^{N}-u\right|_{\mathbb{V}} d s+C E \int_{0}^{\tau_{M}}\left|u^{N}\right|_{\mathbb{W}}\left|u^{N}-u\right|_{\mathbb{V}} d s .
\end{aligned}
$$

We derive from (38) that

$$
\lim _{N \rightarrow \infty} E \int_{0}^{T} 1_{\left[0, \tau_{M}\right]}\left\langle\widehat{B}\left(u, u-u^{N}\right), w(s)\right\rangle-\left\langle\widehat{B}\left(u^{N}, u-u^{N}\right), w(s)\right\rangle d s=0
$$

Since $\tau_{M} \nearrow T$ almost surely and $L^{\infty}\left(\Omega, \mathcal{F}, P ; L^{\infty}(0, T ; \mathbb{W})\right)$ is dense in $L^{2}\left(\Omega, \mathcal{F}, P ; L^{2}(0, T ; \mathbb{W})\right)$, then we deduce from (60)-(63) that

$$
\widehat{B}(u(.), u(.))=\widehat{B}^{*} .
$$

Next, thanks to the property of $\widehat{F}$ we see that

$$
E \int_{0}^{\tau_{M}}\left|\widehat{F}\left(u^{N}\right)-\widehat{F}(u)\right|_{\mathbb{V}}^{2} d s \leq C_{F} E \int_{0}^{\tau_{M}}\left|u^{N}-u\right|_{\mathbb{V}}^{2} d s
$$

Owing to (38) and the fact that $\tau_{M} \nearrow T$ almost surely as $M \rightarrow \infty$, we obtain that

$$
\widehat{F}\left(u^{N}\right) \rightarrow \widehat{F}(u) \text { strongly in } L^{2}\left(\Omega, \mathcal{F}, P ; L^{2}(0, T ; \mathbb{V})\right) .
$$

From (59) we see that

$$
\widehat{G}^{*}(.)=\widehat{G}(u(.))
$$

as an element of $L^{2}\left(\Omega, \mathcal{F}, P ; L^{2}\left(0, T ; \mathbb{V}^{\otimes m}\right)\right)$. This complete the proof of the Lemma 3.8. 
It follows from (64), (65), (66), and (33) that $u$ is a solution of the stochastic model for the second grade fluid. The proof of the Theorem 3.4 is finished.

\section{Uniqueness of the solution and strong convergence of the whole sequence of Galerkin approximation}

As already mentioned in the introduction we also discuss the pathwise uniqueness of solutions. More precisely we prove the following result.

Theorem 4.1. Let $u_{1}$ and $u_{2}$ be two strong solutions defined on the stochastic basis $\left(\Omega, \mathcal{F}, \mathcal{F}^{t}, P\right)$ of the problem (1). If we set $v=u_{1}-u_{2}$, then we have $v=0$ almost surely.

Proof. Let $u_{1}$ and $u_{2}$ be two solutions with the same initial condition $u_{0}$ and let $v=u_{1}-u_{2}$. It can be shown that the process $v$ satisfies the equation

$$
\begin{aligned}
& d(v(t)+\alpha A v(t))+\nu A v(t) d t-F\left(u_{1}(t), t\right)-F\left(u_{2}(t), t\right) d t+G\left(u_{1}(t), t\right)-G\left(u_{2}(t), t\right) d W \\
& =-\mathbb{P}\left(\operatorname{curl}\left(u_{1}(t)-\alpha \Delta u_{1}(t)\right) \times u_{1}(t)-\operatorname{curl}\left(u_{2}(t)-\alpha \Delta u_{2}(t)\right) \times u_{2}(t)\right) d t
\end{aligned}
$$

and $v(0)=0$. Multiplying this equation by $(I+\alpha A)^{-1}$ and applying Itô's formula to $|v|_{\mathbb{V}}^{2}$, we obtain that

$$
\begin{aligned}
& |v(t)|_{\mathbb{V}}^{2}+2 \int_{0}^{t}\left(\nu\|v(s)\|^{2}-\left(\operatorname{curl}(v(s)-\alpha \Delta v(s)) \times v(s), u_{2}(s)\right)\right) d s \\
& =\int_{0}^{t}\left(2\left(\widehat{F}\left(u_{1}(s), s\right)-\widehat{F}\left(u_{2}(s), s\right), v(s)\right)_{\mathbb{V}}+\left|\widehat{G}\left(u_{1}(s), s\right)-\widehat{G}\left(u_{2}(s), s\right)\right|_{\mathbb{V} \otimes m}^{2}\right) d s \\
& +2 \int_{0}^{t}\left(\widehat{G}\left(u_{1}(s), s\right)-\widehat{G}\left(u_{2}(s), s\right), v(s)\right)_{\mathbb{V}} d W .
\end{aligned}
$$

We recall that the operator $(I+\alpha A)^{-1}$ is linear and bijective from $\mathbb{H}$ into $\mathbb{H}^{2} \mathcal{O} \cap \mathbb{V}$. Moreover for any $f \in \mathbb{H}$ we have

$$
\left|(I+\alpha A)^{-1} f\right|_{\mathbb{V}} \leq C|f| .
$$

For $t \in[0, T]$ let us consider the real-valued process $\sigma(t)=\exp \left(-\int_{0}^{t}\left|u_{2}(s)\right|_{\mathbb{W}}^{2} d s\right)$. We apply Itô's formula to $\sigma(t)|v(t)|_{\mathbb{V}}^{2}$ and find that

$$
\begin{aligned}
& \sigma(t)|v(t)|_{\mathbb{V}}^{2}+2 \int_{0}^{t}\left(\sigma(s)\left(\nu|| v(s) \|^{2}-\left(\operatorname{curl}(v(s)-\alpha \Delta v(s)) \times v(s), u_{2}(s)\right)\right) d s\right. \\
& =\int_{0}^{t}\left(\sigma(s)\left(2\left(\widehat{F}\left(u_{1}(s), s\right)-\widehat{F}\left(u_{2}(s), s\right), v(s)\right)_{\mathbb{V}}+\left|\widehat{G}\left(u_{1}(s), s\right)-\widehat{G}\left(u_{2}(s), s\right)\right|_{\mathbb{V} \otimes m}^{2}\right) d s\right. \\
& -\int_{0}^{t} \sigma(s)\left|u_{2}(s)\right|_{\mathbb{W}}^{2}|v(s)|_{\mathbb{V}}^{2} d s+2 \int_{0}^{t} \sigma(s)\left(\widehat{G}\left(u_{1}(s), s\right)-\widehat{G}\left(u_{2}(s), s\right), v(s)\right)_{\mathbb{V}} d W
\end{aligned}
$$

Using the assumptions (17)-(20) together with (11) and (67), we derive from the previous estimate that

$$
\begin{array}{r}
\sigma(t)|v(t)|_{\mathbb{V}}^{2}+\left.\nu \int_{0}^{t} \sigma(s)|| v(s)\right|^{2} d s \leq C \\
\int_{0}^{t} \sigma(s)|v(s)|_{\mathbb{V}}^{2} d s+2 C \int_{0}^{t} \sigma(s)|v(s)|_{\mathbb{V}}^{2}\left|u_{2}(s)\right|_{\mathbb{W}} d s \\
+2 \int_{0}^{t} \sigma(s)\left(\widehat{G}\left(u_{1}(s), s\right)-\widehat{G}\left(u_{2}(s), s\right), v(s)\right)_{\mathbb{V}} d W \\
-\int_{0}^{t} \sigma(s)\left|u_{2}(s)\right|_{\mathbb{W}}^{2}|v(s)|_{\mathbb{V}}^{2} d s .
\end{array}
$$


For any $\varepsilon>0$, we have

$$
C|v(s)|_{\mathbb{V}}^{2}\left|u_{2}(s)\right|_{\mathbb{W}} \leq C_{\varepsilon}|v(s)|_{\mathbb{V}}^{2}+C^{2} \varepsilon|v(s)|_{\mathbb{V}}^{2}\left|u_{2}(s)\right|_{\mathbb{W}}^{2},
$$

for $0 \leq s \leq t$. We infer from this and (68) that

$$
\begin{array}{r}
\sigma(t)|v(t)|_{\mathbb{V}}^{2}+\left.\nu \int_{0}^{t} \sigma(s)|| v(s)\right|^{2} d s \leq C \int_{0}^{t} \sigma(s)|v(s)|_{\mathbb{V}}^{2} d s+2 \varepsilon C^{2} \int_{0}^{t} \sigma(s)|v(s)|_{\mathbb{V}}^{2}\left|u_{2}(s)\right|_{\mathbb{W}}^{2} d s \\
+2 \int_{0}^{t} \sigma(s)\left(\widehat{G}\left(u_{1}(s), s\right)-\widehat{G}\left(u_{2}(s), s\right), v(s)\right)_{\mathbb{V}} d W \\
-\int_{0}^{t} \sigma(s)\left|u_{2}(s)\right|_{\mathbb{W}}^{2}|v(s)|_{\mathbb{V}}^{2} d s .
\end{array}
$$

Choosing $\varepsilon=\frac{2}{C^{2}}$, we obtain

$$
\sigma(t)|v(t)|_{\mathbb{V}}^{2} \leq C \int_{0}^{t} \sigma(s)|v(s)|_{\mathbb{V}}^{2} d s+2 \int_{0}^{t} \sigma(s)\left(\widehat{G}\left(u_{1}(s), s\right)-\widehat{G}\left(u_{2}(s), s\right), v(s)\right)_{\mathbb{V}} d W .
$$

We get rid of the stochastic term by taking the mathematical expectation. This enables us to write

$$
E \sigma(t)|v(t)|_{\mathbb{V}}^{2} \leq C \int_{0}^{t} E \sigma(s)|v(s)|_{\mathbb{V}}^{2} d s
$$

and the proof of Theorem 4.1 follows from the application of Gronwall's inequality.

Next, we will show that the whole sequence of the Galerkin approximation converges in mean square to the strong probabilistic solution.

Theorem 4.2. The whole sequence of Galerkin approximations $\left(u^{N}\right)_{N \geq 1}$ defined by $(26)$ satisfies

$$
\begin{array}{r}
\lim _{N \rightarrow \infty} E\left|u^{N}(t)-u(t)\right|_{\mathbb{V}}^{2}=0, \\
\lim _{N \rightarrow \infty} E \int_{0}^{T}\left|u^{N}(t)-u(t)\right|_{\mathbb{V}}^{2} d t=0,
\end{array}
$$

where $u$ is the strong probabilistic solution of (1).

The main ingredient of the proof of this result is the following lemma, its proof can be found in [9].

Lemma 4.3. Let $\left\{Q_{N} ; N \geq 1\right\} \subset L^{2}\left(\Omega, \mathbb{P}, L^{2}(0, T ; \mathbb{R})\right)$ be a sequence of continuous realvalued process, and let $\left\{\sigma_{M} ; M \geq 1\right\}$ be a sequence of $\mathcal{F}^{t}$-stopping times such that $\sigma_{M}$ is increasing to $T, \sup _{n>1} \mathbb{E}\left|Q_{N}(T)\right|^{2}<\infty$, and $\lim _{N \rightarrow \infty} \mathbb{E}\left|Q_{N}\left(\sigma_{M}\right)\right|=0$ for all $M \geq 1$. Then $\lim _{N \rightarrow \infty} \mathbb{E}\left|Q_{N}(\bar{T})\right|=0$.

Proof of Theorem 4.2. It follows from (59) and (40) that

$$
\lim _{N \rightarrow \infty} \mathbb{E} \int_{0}^{\tau_{M}}\left|u^{N}(t)-u(t)\right|_{\mathbb{V}}^{2} d t=0
$$

and

$$
\lim _{N \rightarrow \infty} \mathbb{E}\left|u^{N}\left(\tau_{M}\right)-u\left(\tau_{M}\right)\right|_{\mathbb{V}}^{2}=0
$$

for any $M \geq 1$. So by applying the preceding lemma to $Q_{N}(t)=\int_{0}^{t}\left|u^{N}(s)-u(s)\right|_{\mathbb{V}}^{2} d s$ (resp., $\left.Q_{N}(t)=\left|u^{N}(t)-u(t)\right|_{\mathbb{V}}^{2}\right), \sigma_{M}=\tau_{M}$ and taking into account (71)-(72), the estimates in Lemma 3.7 and the uniqueness of $u$, we see that the whole sequence $u^{N}$ defined by (26) satisfies (69) and (70). This ends the proof of Theorem 4.2. 


\section{Analysis of the asymptotic behaviour of the solutions}

In this last section we briefly discuss the long time behaviour of the strong solutions of the stochastic model for the second grade fluids, we will mainly follow the idea in [13] and [15]. We consider two subsections. In the first one, we study the exponential decay in mean square of the strong solution. In the second, we strengthen the assumption on the external force $F$ and investigate the exponential stability in mean square of the non-trivial stationary solution.

\subsection{The exponential decay of the strong solution}

We require that $u_{0}$ (resp. $F$ and $G$ ) satisfies the assumptions in Remark 3.5 (resp. Remark 3.1). We suppose furthermore that there exist $\theta>0, M_{\beta}>0$ such that

$$
0<|F(0, t)|_{\mathbb{V}} \leq M_{\beta} e^{-\theta t}, \forall t>0,
$$

and

$$
|F(u, t)-F(v, t)|_{\mathbb{V}} \leq\left(C_{F}+\beta(t)\right)|u-v|_{\mathbb{V}}, \quad \forall t>0, \text { and } u, v \in \mathbb{V} .
$$

The function $\beta(t)$ is an integrable function such that $\beta(t)=O\left(e^{-\theta t}\right)$. Hereafter given two real-valued functions $f$ and $g$, we say that $f(t)=O(g(t))$ if there exists $C>0$ and $t_{0} \geq 0$ such that $|f(t)| \leq C|g(t)|$ for all $t>t_{0}$.

We also assume that

$$
0<|G(0, t)|_{\mathbb{V} \otimes m} \leq M_{\delta} e^{-\theta t}, \forall t>0
$$

and

$$
|G(u, t)-G(v, t)|_{\mathbb{V} \otimes m} \leq(\eta+\delta(t))|u-v|_{\mathbb{V}}, \quad \forall t>0, \text { and } u, v \in \mathbb{V} .
$$

Here $\delta(t)$ is an integrable function such that $\delta(t)=O\left(e^{-\theta t}\right)$. Before we formulate and prove the main result of this subsection we introduce the following

Definition 5.1. The strong probabilistic solution $u$ of (23) is said to decay exponentially in mean square if there exist positive constants $a$ and $b$ such that

$$
E|u(t)|_{\mathbb{V}}^{2} \leq b e^{-a t}
$$

for $t$ large enough.

We have

Theorem 5.2. Assume that

$$
\frac{2 \nu}{\mathcal{P}^{2}+\alpha}>\frac{2 \mathcal{P}^{2} C_{F}}{\alpha}+\frac{2 \mathcal{P}^{4} \eta}{\alpha^{2}}+1
$$

then there exists $a \in(0, \theta)$ such that

$$
E|u(t)|_{\mathbb{V}}^{2} \leq\left(E\left|u_{0}\right|_{\mathbb{V}}^{2}+\frac{4 \alpha^{2} M_{\beta}+2 \mathcal{P}^{4} M_{\delta}}{\alpha^{2}(\theta-a)}\right) e^{-a t}, \forall t>0 .
$$

Proof. Since

$$
\frac{2 \nu}{\mathcal{P}^{2}+\alpha}>\frac{2 \mathcal{P}^{2} C_{F}}{\alpha}+\frac{2 \mathcal{P}^{4} \eta}{\alpha^{2}}+1
$$


we can choose $a \in(0, \theta)$ such that

$$
\frac{2 \nu}{\mathcal{P}^{2}+\alpha} \geq a+\frac{2 \mathcal{P}^{2} C_{F}}{\alpha}+\frac{2 \mathcal{P}^{4} \eta}{\alpha^{2}}+1
$$

It follows from the application of Itô's formula that

$$
\begin{aligned}
e^{a t}|u(t)|_{\mathbb{V}}^{2} & =\left|u_{0}\right|_{\mathbb{V}}^{2}-2 \int_{0}^{t} e^{a s} \nu \|\left. u\right|^{2}+2 \int_{0}^{t} e^{a s}(\widehat{F}(u, s), u)_{\mathbb{V}} d s+\int_{0}^{t} e^{a s}|\widehat{G}(u, s)|_{\mathbb{V} \otimes m} d s \\
& +a \int_{0}^{t} e^{a s}|u(s)|_{\mathbb{V}}^{2} d s+2 \int_{0}^{t}(\widehat{G}(u, s), u)_{\mathbb{V}} d W .
\end{aligned}
$$

Taking the mathematical expectation and using the property of the stochastic integral and Cauchy-Schwarz's inequality we get

$$
\begin{aligned}
e^{a t} E|u(t)|_{\mathbb{V}}^{2} & \leq E\left|u_{0}\right|_{\mathbb{V}}^{2}-\frac{2 \nu}{\mathcal{P}^{2}+\alpha} \int_{0}^{t} e^{a s} E|u(s)|_{\mathbb{V}}^{2} d s+\left.2 \int_{0}^{t} e^{a s} E|\widehat{F}(u, s)|\right|_{\mathbb{V}}|u|_{\mathbb{V}} d s \\
& +\int_{0}^{t} e^{a s} E|\widehat{G}(u, s)|_{\mathbb{V} \otimes m} d s+a \int_{0}^{t} e^{a s} E|u(s)|_{\mathbb{V}}^{2} d s .
\end{aligned}
$$

Invoking the estimate (77) and making use of the assumptions on $F$ and $G$ yield

$$
\begin{aligned}
e^{a t} E|u(t)|_{\mathbb{V}}^{2} & \leq E\left|u_{0}\right|_{\mathbb{V}}^{2}+\left(a-\frac{2 \nu}{\mathcal{P}^{2}+\alpha}+2 \frac{\mathcal{P}^{2}}{\alpha}+2 \frac{\mathcal{P}^{4}}{\alpha^{2}}+1\right) \int_{0}^{t} e^{a s} E|u(s)|_{\mathbb{V}}^{2} d s \\
& +\int_{0}^{t}\left(2 \frac{\mathcal{P}^{2}}{\alpha} \beta(s)+2 \frac{\mathcal{P}^{4}}{\alpha^{2}} \delta(s)\right) e^{a s} E|u(s)|_{\mathbb{V}}^{2} d s \\
& +\int_{0}^{t}\left(4 M_{\beta}+2 \frac{\mathcal{P}^{4}}{\alpha^{2}} M_{\delta}\right) e^{(a-\theta) s} d s .
\end{aligned}
$$

The application of Gronwall's inequality to this estimate implies

$$
\begin{aligned}
e^{a t} E|u(t)|_{\mathbb{V}}^{2} & \leq \exp \left\{\left(1+a+2 \frac{\mathcal{P}^{2}}{\alpha}+2 \frac{\mathcal{P}^{4}}{\alpha^{2}}-\frac{2 \nu}{\mathcal{P}^{2}+\alpha}\right) t\right\} \exp \left\{\int_{0}^{t}\left(2 \frac{\mathcal{P}^{2}}{\alpha} \beta(s)+2 \frac{\mathcal{P}^{4}}{\alpha^{2}} \delta(s)\right) d s\right\} \\
& \times\left(E\left|u_{0}\right|_{\mathbb{V}}^{2}+\frac{4 \alpha^{2} M_{\beta}+2 \mathcal{P}^{4} M_{\delta}}{\alpha^{2}(a-\theta)}\left(e^{(a-\theta) t}-1\right)\right) .
\end{aligned}
$$

With the choice of $a$ and the assumption on $\beta(s)$ and $\delta(s)$ we obtain that

$$
E|u(t)|_{\mathbb{V}}^{2} \leq E\left|u_{0}\right|_{\mathbb{V}}^{2} e^{-a t}+\frac{4 \alpha^{2} M_{\beta}+2 \mathcal{P}^{4} M_{\delta}}{\alpha^{2}(\theta-a)} e^{-a t}, \quad \forall t>0
$$

This completes the proof of the theorem.

\subsection{The stability of the stationary solution}

In this subsection we strengthen the assumptions on $F$ by requiring that $F(u, t)=F(u)$ for any $t \in[0, T]$ and

$$
\begin{gathered}
F(0) \neq 0, \\
\|F(u)-F(v)\| \leq C_{F}\|u-v\|,
\end{gathered}
$$

We associate to (23) the deterministic equation

$$
u(t)+\int_{0}^{t}(\nu \widehat{A} u+\widehat{B}(u)) d s=u_{0}+\int_{0}^{t} \widehat{F}(u) d s .
$$


Definition 5.3. The function $u_{\infty}$ is said to be a stationary solution of (73) if it satisfies the time-independent problem

$$
\left\{\begin{array}{l}
-\nu \Delta u_{\infty}+\operatorname{curl}\left(u_{\infty}-\alpha \Delta u_{\infty}\right) \times u_{\infty}+\nabla \mathfrak{P}=F\left(u_{\infty}\right) \text { in } D \\
\operatorname{div} u_{\infty}=0 \text { in } D \\
u_{\infty}=0 \text { on } \partial D
\end{array}\right.
$$

Or equivalently, it verifies the following

$$
\nu \widehat{A} u_{\infty}+\widehat{B}\left(u_{\infty}, u_{\infty}\right)=\widehat{F}\left(u_{\infty}\right)
$$

as an equation in $\mathbb{W}^{*}$.

The analysis of (74) was done in [6] for the 3-dimensional case. The method in [6], which is a combination of the Galerkin method and the Brouwer fixed point method yields the following result.

Theorem 5.4. Let $\alpha>0, \nu>0$ such that

$$
\nu-\mathcal{P} C_{F}>0 .
$$

Then, there exists a solution $u_{\infty}$ of (74). This function $u_{\infty}$ verifies

$$
\left\|u_{\infty}\right\| \leq \frac{\mathcal{P}^{2}}{\nu-\mathcal{P}^{2} C_{F}}\|F(0)\|,
$$

and

$$
\left|u_{\infty}\right| \mathbb{w} \leq K(\alpha, \nu, F)
$$

where $K(\alpha, \nu, F)$ is equal to

$$
\sqrt{2}\|F(0)\|\left[\frac{\mathcal{P}^{2}}{\nu-\mathcal{P}^{2} C_{F}}\left(\frac{\alpha C_{F}}{\nu}+1\right)+1\right] .
$$

Furthermore, this solution is unique provided that

$$
\nu-C_{B} K(\alpha, \nu, F)-\mathcal{P}^{2} C_{f}>0 .
$$

Definition 5.5. The stationary solution $u_{\infty}$ of (73) is said to be exponentially stable in mean square if there are positive constants $a$ and $b$ such that

$$
E\left|u(t)-u_{\infty}\right|_{\mathbb{V}}^{2} \leq b e^{-a t},
$$

for any $t>0$.

In this definition $u(t)$ is the strong solution of $(23)$ and the term strong solution should be understood in the sense of Definition 3.2.

We prove the following

Theorem 5.6. For any $\theta>0$, assume that $G$ satisfies

$$
\left|G\left(u_{\infty}, t\right)\right|_{\mathbb{V} \otimes m}^{2} \leq \eta e^{-\theta t}
$$

and

$$
|G(u, t)-G(v, t)|_{\mathbb{V} \otimes m}^{2} \leq \delta(t)|u-v|_{\mathbb{V}}^{2}, \quad \forall(u, v) \in \mathbb{V} \times \mathbb{V}
$$


where $\delta(t)$ is a positive integrable function such that $\delta(t) \leq C_{\delta} e^{-\theta t}, C_{\delta}>0$. Assume also that

$$
\nu>2 C_{B}\left|u_{\infty}\right| \mathbb{W}+\frac{\mathcal{P}^{2}}{\alpha} .
$$

Then there exists a constant $a \in(0, \theta)$ such that the following holds

$$
E\left|u(t)-u_{\infty}\right|_{\mathbb{V}}^{2} \leq\left(E\left|u_{0}-u_{\infty}\right|_{\mathbb{V}}^{2}+\frac{\eta}{(\theta-a)}\right) e^{-a t}, \forall t>0 .
$$

Proof. Thanks to Itô's formula, we have

$$
\begin{aligned}
\left|u(t)-u_{\infty}\right|_{\mathbb{V}}^{2} & =\left|u_{0}-u_{\infty}\right|_{\mathbb{V}}^{2}-2 \int_{0}^{t}\left[\left(\nu A u, u-u_{\infty}\right)+\left\langle\widehat{B}(u, u), u-u_{\infty}\right\rangle\right] d s \\
& +2 \int_{0}^{t}\left(F(u), u-u_{\infty}\right) d s+\int_{0}^{t}|\widehat{G}(u, s)|_{\mathbb{V} \otimes m} d s \\
& +2 \int_{0}^{t}\left(\widehat{G}(u, s), u-u_{\infty}\right)_{\mathbb{V}} d W .
\end{aligned}
$$

Similarly

$$
\begin{aligned}
e^{a t}\left|u(t)-u_{\infty}\right|_{\mathbb{V}}^{2} & =\left|u_{0}-u_{\infty}\right|_{\mathbb{V}}^{2}-2 \int_{0}^{t} e^{a s}\left[\left(\nu A u, u-u_{\infty}\right)+\left\langle\widehat{B}(u, u), u-u_{\infty}\right\rangle\right] d s \\
& +2 \int_{0}^{t} e^{a s}\left(F(u), u-u_{\infty}\right) d s+a \int_{0}^{t} e^{a s}\left|u-u_{\infty}\right|_{\mathbb{V}}^{2} d s \\
& +\int_{0}^{t} e^{a s}|\widehat{G}(u, s)|_{\mathbb{V} \otimes m} d s+2 \int_{0}^{t} e^{a s}\left(\widehat{G}(u, s), u-u_{\infty}\right)_{\mathbb{V}} d W
\end{aligned}
$$

for $a>0$. Taking the mathematical expectation in both sides of this equation, and using property of stochastic integrals we obtain

$$
\begin{aligned}
e^{a t} E\left|u(t)-u_{\infty}\right|_{\mathbb{V}}^{2} & =E\left|u_{0}-u_{\infty}\right|_{\mathbb{V}}^{2}-2 \int_{0}^{t} e^{a s} E\left[\left(\nu A u, u-u_{\infty}\right)+\left\langle\widehat{B}(u, u), u-u_{\infty}\right\rangle\right] d s \\
& +2 \int_{0}^{t} e^{a s} E\left(F(u), u-u_{\infty}\right) d s+\int_{0}^{t} e^{a s} E|\widehat{G}(u, s)|_{\mathbb{V} \otimes m} d s \\
& +a \int_{0}^{t} e^{a s} E\left|u-u_{\infty}\right|_{\mathbb{V}}^{2} d s
\end{aligned}
$$

Since $u_{\infty}$ is a solution of $(74)$, then we have

$$
\int_{0}^{t} e^{a s} E\left[\left(\nu A u_{\infty}, u-u_{\infty}\right)+\left\langle\widehat{B}\left(u_{\infty}, u_{\infty}\right), u-u_{\infty}\right\rangle\right] d s=\int_{0}^{t} e^{a s} E\left(F\left(u_{\infty}\right), u-u_{\infty}\right) d s .
$$

We deduce from the last two equations that

$$
\begin{gathered}
e^{a t} E\left|u(t)-u_{\infty}\right|_{\mathbb{V}}^{2}=E\left|u_{0}-u_{\infty}\right|_{\mathbb{V}}^{2}+a \int_{0}^{t} e^{a s} E\left|u-u_{\infty}\right|_{\mathbb{V}}^{2} d s-2 \nu \int_{0}^{t} e^{a s} E|| u-u_{\infty}||^{2} d s \\
-2 \int_{0}^{t} e^{a s}\left\langle\widehat{B}(u, u)-\widehat{B}\left(u_{\infty}, u_{\infty}\right), u-u_{\infty}\right\rangle d s+\int_{0}^{t} e^{a s} E|\widehat{G}(u, s)|_{\mathbb{V} \otimes m} d s \\
+2 \int_{0}^{t} e^{a s} E\left(F(u)-F\left(u_{\infty}\right), u-u_{\infty}\right) d s
\end{gathered}
$$


By Cauchy-Schwarz's inequality it follows from this relation that

$$
\begin{gathered}
e^{a t} E\left|u(t)-u_{\infty}\right|_{\mathbb{V}}^{2} \leq E\left|u_{0}-u_{\infty}\right|_{\mathbb{V}}^{2}+a \int_{0}^{t} e^{a s} E\left|u-u_{\infty}\right|_{\mathbb{V}}^{2} d s-2 \nu \int_{0}^{t} e^{a s} E|| u-u_{\infty} \|^{2} d s \\
-2 \int_{0}^{t} e^{a s}\left\langle\widehat{B}(u, u)-\widehat{B}\left(u_{\infty}, u_{\infty}\right), u-u_{\infty}\right\rangle d s+\int_{0}^{t} e^{a s} E|\widehat{G}(u, s)|_{\mathbb{V} \otimes m} d s \\
+2 \int_{0}^{t} e^{a s} E\left[\mid F(u)-F\left(u_{\infty}|| u-u_{\infty} \mid\right] d s .\right.
\end{gathered}
$$

But

$$
\left\langle\widehat{B}(u, u)-\widehat{B}\left(u_{\infty}, u_{\infty}\right), u-u_{\infty}\right\rangle=\left\langle\widehat{B}\left(u-u_{\infty}, u\right), u-u_{\infty}\right\rangle
$$

and

$$
\left\langle\widehat{B}(u, u)-\widehat{B}\left(u_{\infty}, u_{\infty}\right), u-u_{\infty}\right\rangle=-\left\langle\widehat{B}\left(u-u_{\infty}, u-u_{\infty}\right), u_{\infty}\right\rangle,
$$

then we can infer from these and (75) that

$$
\begin{array}{r}
e^{a t} E\left|u(t)-u_{\infty}\right|_{\mathbb{V}}^{2} \leq E\left|u_{0}-u_{\infty}\right|_{\mathbb{V}}^{2}+a \int_{0}^{t} e^{a s} E\left|u-u_{\infty}\right|_{\mathbb{V}}^{2} d s-2 \nu \int_{0}^{t} e^{a s} E|| u-u_{\infty}||^{2} d s \\
+2 \int_{0}^{t} e^{a s}\left\langle\widehat{B}\left(u-u_{\infty}, u-u_{\infty}\right), u_{\infty}\right\rangle d s+\int_{0}^{t} e^{a s} E|\widehat{G}(u, s)|_{\mathbb{V} \otimes m} d s \\
+2 \int_{0}^{t} e^{a s} E\left[\mid F(u)-F\left(u_{\infty}|| u-u_{\infty} \mid\right] d s .\right.
\end{array}
$$

By (11) and the assumption on $F$ we see from the previous estimate that

$$
\begin{array}{r}
e^{a t} E\left|u(t)-u_{\infty}\right|_{\mathbb{V}}^{2} \leq E\left|u_{0}-u_{\infty}\right|_{\mathbb{V}}^{2}+a \int_{0}^{t} e^{a s} E\left|u-u_{\infty}\right|_{\mathbb{V}}^{2} d s-\frac{2 \nu}{\mathcal{P}^{2}+\alpha} \int_{0}^{t} e^{a s} E\left|u-u_{\infty}\right|_{\mathbb{V}}^{2} d s \\
+2 C_{B} \int_{0}^{t} e^{a s} E\left[\left|u-u_{\infty}\right|_{\mathbb{V}}^{2}\left|u_{\infty}\right|_{\mathbb{W}}\right] d s+\int_{0}^{t} e^{a s} E|\widehat{G}(u, s)|_{\mathbb{V} \otimes m} d s \\
+2 \frac{\mathcal{P}^{2} C_{F}}{\alpha} \int_{0}^{t} e^{a s} E\left|u-u_{\infty}\right|_{\mathbb{V}}^{2} d s .
\end{array}
$$

It is not hard to show that

$$
\left|(I+\alpha A)^{-1} v\right|_{\mathbb{V}}^{2} \leq \frac{\mathcal{P}^{2}}{\alpha}|v|^{2} \leq \frac{\mathcal{P}^{4}}{\alpha^{2}}|v|_{\mathbb{V}}^{2},
$$

for any $v \in \mathbb{V}$. We use this estimate in (76) and obtain

$$
\begin{array}{r}
e^{a t} E\left|u(t)-u_{\infty}\right|_{\mathbb{V}}^{2} \leq E\left|u_{0}-u_{\infty}\right|_{\mathbb{V}}^{2}+\left(a+2 C_{B}\left|u_{\infty}\right|_{\mathbb{W}}+\frac{\mathcal{P}^{2} C_{F}}{\alpha}-2 \nu\right) \int_{0}^{t} e^{a s} E\left|u-u_{\infty}\right|_{\mathbb{V}}^{2} d s \\
+\frac{\mathcal{P}^{4}}{\alpha^{2}} \int_{0}^{t} e^{a s} E|G(u, s)|_{\mathbb{V}}^{2} d s .
\end{array}
$$

The assumptions on $G$ give

$$
\begin{array}{r}
e^{a t} E\left|u(t)-u_{\infty}\right|_{\mathbb{V}}^{2} \leq E\left|u_{0}-u_{\infty}\right|_{\mathbb{V}}^{2}+\int_{0}^{t}\left(a+2 C_{B}\left|u_{\infty}\right| \mathbb{W}\right. \\
\left.+\frac{\mathcal{P}^{2} C_{F}}{\alpha}-2 \nu\right) e^{a s} E\left|u-u_{\infty}\right|_{\mathbb{V}}^{2} d s \\
\left.+2 \frac{\mathcal{P}^{4}}{\alpha^{2}} \int_{0}^{t} \delta(s)\right) e^{a s} E\left|u-u_{\infty}\right|_{\mathbb{V}}^{2} d s \\
+2 \frac{\mathcal{P}^{4}}{\alpha^{2}} \eta \int_{0}^{t} e^{(a-\theta) s} d s
\end{array}
$$


Using Gronwall's inequality in the above estimate, we obtain

$$
\begin{aligned}
e^{a t} E\left|u(t)-u_{\infty}\right|_{\mathbb{V}}^{2} \leq \exp \left\{\left(a+2 C_{B}\left|u_{\infty}\right|_{\mathbb{W}}\right.\right. & \left.\left.+\frac{\mathcal{P}^{2} C_{F}}{\alpha}-2 \nu\right) t\right\} \exp \left\{2 \frac{\mathcal{P}^{4}}{\alpha^{2}} \int_{0}^{t} \delta(s) d s\right\} \\
& \times\left(E\left|u_{0}-u_{\infty}\right|_{\mathbb{V}}^{2}+\frac{\eta}{a-\theta}\left(e^{(a-\theta) t}-1\right) d s\right),
\end{aligned}
$$

for any $t>0$. Since

$$
2 C_{B}\left|u_{\infty}\right| \mathbb{W}+\frac{\mathcal{P}^{2} C_{F}}{\alpha}<2 \nu,
$$

then we may choose $a \in(0, \theta)$ such that

$$
a+2 C_{B}\left|u_{\infty}\right| \mathbb{w}+\frac{\mathcal{P}^{2} C_{F}}{\alpha} \leq 2 \nu .
$$

With this choice and the assumption on $\delta$ we have

$$
E\left|u(t)-u_{\infty}\right|_{\mathbb{V}}^{2} \leq\left(E\left|u_{0}-u_{\infty}\right|_{\mathbb{V}}^{2}+\frac{\eta}{(\theta-a)}\right) e^{-a t}, \forall t>0 .
$$

And this completes the proof of the theorem.

\section{Acknowledgment}

The research of the authors is supported by the University of Pretoria and the National Research Foundation South Africa. The first author is also grateful to the Abdus Salam ICTP, Trieste, Italy for its hospitality. The second author was supported by the National Science Foundation grant No DMS-0635607 during his stay at IAS, Princeton.

\section{References}

[1] R. A. Adams. Sobolev Spaces. Academic Press, 1975.

[2] S. Albeverio, Z. Brzezniak and J.-L. Wu. Existence of global solutions and invariant measures for stochastic differential equations driven by Poisson type noise with nonLipschitz coefficients. J. Math. Anal. Appl. 371(1):309-322, 2010.

[3] A. Bensoussan. Stochastic Navier-Stokes Equations. Acta Applicandae Mathematicae, 38:267-304, 1995.

[4] A. Bensoussan and R. Temam. Equations Stochastiques du Type Navier-Stokes. Journal of Functional Analysis, 13:195-222, 1973.

[5] J. M. Bernard. Weak and Classical Solutions of equations of Motion for Second Grade Fluids. Comm. Appl. Nonlinear Anal., 5(4):1-32, 1998.

[6] J. M. Bernard. Stationary Problem of Second-grade Fluids in Three Dimensions: Existence, Uniqueness and Regularity. Math. Meth. Appl. Sci., 22: 655-687, 1999.

[7] A. Bonito, P. Clément, and M. Picasso Mathematical analysis of a simplified Hookean dumbbells model arising from viscoelastic flows. J. Evol. Equ., 6:381-398, 2006. 
[8] H. Breckner Galerkin approximation and the strong solution of the Navier-Stokes equation. Journal of Applied Mathematics and Stochastic Analysis. 13(3):239-259, 2000.

[9] H. Breckner Approximation and optimal control of the stochastic navier-Stokes equation Dissertation, Martin-Luther University, Halle-Wittenberg, 1999.

[10] Z. Brzezniak and L. Debbi. On stochastic Burgers equation driven by a fractional Laplacian and space-time white noise. Stochastic differential equations: Theory and applications, Interdiscip. Math. Sci., 2, World Sci. Publ., pages 135-167, 2007.

[11] Z. Brzezniak, B. Maslowski and J. Seidler. Stochastic nonlinear beam equation, Probab. Theory Relat. Fields. 132(2):119-144, 2005.

[12] V. Busuioc. On second grade fluids with vanishing viscosity. C. R. Acad. Paris, Série I, 328(12):1241-1246, 1999.

[13] T. Caraballo, J.A. Langa and T. Taniguchi. The exponential behaviour and stabilizability of stochastic 2D-Navier-Stokes equations. J. Differential Equations. 179(2):714-737, 2002.

[14] T. Caraballo, J. Real and T. Taniguchi. On the exisrence and uniqueness of solutions to stochastic three-dimensional Lagrangian averaged Navier-Stokes equations. Proc. R. Soc. Lond. Ser. A Math. Phys. Eng. Sci. Vol 462 No 2066:459-479, 2006.

[15] T. Caraballo, A.M. Márquez-Durán and J. Real. The asymptotic behaviour of a stochastic 3D LANS- $\alpha$ model. Appl. Math. Optim. 53(2):141-161, 2006.

[16] D. Cioranescu and V. Girault. Weak and classical solutions of a family of second grade fluids. Int. J. Non-Linear Mechanics, 32(2):317-335, 1997.

[17] D. Cioranescu and E. H. Ouazar. Existence and uniqueness for fluids of second grade. In Nonlinear Partial Differential Equations, Collège de France Seminar, Pitman 109, pages 178-197. 1984.

[18] D. Cioranescu and E. H. Ouazar. Existence et unicité pour les fluides de grade deux. C. R. Acad. Sci. Paris, Série I, 298(13):285-287, 1984.

[19] G. Da Prato and J. Zabczyk. Stochastic Equations in Infinite Dimensions. Cambridge University Press, 1992.

[20] G. Da Prato and A. Debussche 2D stochastic Navier-Stokes equations with a timeperiodic forcing term. J. Dynam. Differential Equations 20(2):301-335, 2008.

[21] G. Deugoue and M. Sango. On the Stochastic 3D Navier-Stokes- $\alpha$ Model of Fluids Turbulence, Abstract and Applied Analysis, vol. 2009, Article ID 723236, 27 pages, 2009. doi:10.1155/2009/723236.

[22] G. Deugoue and M. Sango. On the Strong Solution for the 3D Stochastic LerayAlpha Model, Boundary Value Problems, vol. 2010, Article ID 723018, 31 pages, 2010. doi:10.1155/2010/723018.

[23] J. E. Dunn and R. L. Fosdick. Thermodynamics, stability and boundedness of fluids of complexity two and fluids of second grade. Arch. Rat. Mech. Anal., 56(3):191-252, 1974. 
[24] J. E. Dunn and K. R. Rajagopal Fluids of differntial type: Critical review and thermodynamic analysis. Int. J. Engng. Sci., 33(5): 668-729, 1995

[25] R. L. Fosdick and K.R. Rajagopal Anomalous features in the model of second grade fluids Arch. Rational mech. Analysis, 70:145-152, 1978.

[26] D. D. Holm, J. E. Marsden, and T. Ratiu. The Euler-Poincaré equations and semidirect products with applications to continuum theories. Advances in Math., 137:1-81, 1998.

[27] D. D. Holm, J. E. Marsden, and T. Ratiu. Euler-Poincaré models of ideal fluids with nonlinear dispersion. Phys. Rev. Lett., 349:4173-4177, 1998.

[28] D. Iftimie. Remarques sur la limite $\alpha \rightarrow 0$ pour les fluides de grade 2. C. R. Acad. Sci. Paris, Ser. I, 334(1):83-86, 2002.

[29] B. Jourdain and T. Lelièvre Mathematical analysis of a stochastic differential equation arising in the micro-macro modelling of polymeric fluids. Probabilistic Methods in Fluids Proceedings of the Swansea 2002 Workshop. Davies, I.M., Jacob, N., Truman, A., Hassan, O., Morgan, K.,Weatherill, N.P. (eds.),World Scientific, pp. 205-223, 2003.

[30] B. Jourdain, T. Lelièvre, and C. Le Bris. Existence of solution for a micro-macro model of polymeric fluid: the FENE model. J. Funct. Anal. 209:162-193, 2004.

[31] B. Jourdain, C. Le Bris, T. Lelièvre, and F. Otto. Long-Time Asymptotics of a Multiscale Model for Polymeric Fluid Flows. Arch. Rational Mech. Anal., 181:97$148,2006$.

[32] I. Karatzas and S. E. Shreve. Brownian Motion and Stochastic Calculus. GTM. Springer-Verlag, 1987.

[33] R. Mikulevicius and B.L. Rozovskii. Stochastic Navier-Stokes Equations and Turbulent Flows. SIAM J. Math. Anal., 35(5):1250-1310, 2004.

[34] W. Noll and C. Truesdell. The Nonlinear Field Theory of Mechanics, Volume III of Handbuch der Physik. Springer-Verlag, Berlin, 1975.

[35] A. P. Oskolkov Solvability in the large of the first boundary-value problem for a quasilinear third-order system pertaining to the motion of a viscuous fluid Journal of Soviet Mathematics 3(4):508-521, 1975.

[36] A. P. Oskolkov The uniqueness and gloal solvability of boundary-value problems for the equations of motions for acqueous solutions of polymers Journal of Soviet Mathematics 8(4):427-455, 1977.

[37] H.C. Öttinger. Stochastic processes in polymeric fluids. Springer-Verlag, Berlin, 1996.

[38] E. Pardoux. Equations aux dérivées partielles stochastiques monotones. Thèse de Doctorat, Université Paris-Sud, 1975.

[39] S. Peszat and J. Zabczyk. Stochastic Partial Differential Equations with Levy Noise. An evolution equation approach. Encyclopedia of Mathematics and its Applications 113, Cambridge university Press, 2007. 
[40] P.A. Razafimandimby, Some mathematical problems in the dynamics of stochastic second-grade fluids. Ph.D. Thesis, University of Pretoria, 2010.

[41] P. A. Razafimandimby and M. Sango. Weak Solutions of a Stochastic Model for TwoDimensional Second Grade Fluids, Boundary Value Problems, vol. 2010, Article ID 636140, 47 pages, 2010. doi:10.1155/2010/636140.

[42] P. A. Razafimandimby. On Stochastic Models Describing the Motions of Randomly Forced Linear Viscoelastic Fluids, Journal of Inequalities and Applications, vol. 2010, Article ID 932053, 27 pages, 2010. doi:10.1155/2010/932053.

[43] P.A. Razafimandimby and M. Sango. Asymptotic behaviour of solutions of stochastic evolution equations for second grade fluids, In press C. R. Acad. Sci. Paris, Ser. I (2010), doi:10.1016/j.crma.2010.05.001

[44] M. Sango. Weak solutions for a doubly degenerate quasilinear parabolic equation with random forcing. Discrete Contin. Dyn. Syst. Ser. B, 7(4) (2007) 885-905.

[45] M. Sango. Magnetohydrodynamic turbulent flows: Existence results. Physica D: Nonlinear Phenomena 239(12): 912-923, 2010.

[46] M. Sango. Density dependent stochastic Navier-Stokes equations with non Lipschitz random forcing. Reviews in Mathematical Physics 22(6):669-697, 2010.

[47] S. Shkoller. Geometry and curvature of diffeomorphism groups with $H^{1}$ metric and hydrodynamics. J. Func. Anal., 160:337-365, 1998.

[48] S. Shkoller. Smooth global Lagrangian flow for the 2D Euler and second-grade fluid equations. Appl. Math. Lett., 14:539-543, 2001.

[49] A. V. Skorokhod. Studies in the Theory of Random Processes. Addison-Wesley Publishing Company, 1965.

[50] V. A. Solonnikov. On general boundary problems for systems which are elliptic in the sense of A. Douglis and L. Nirenberg. I. American Mathematical Society Translations, 56:193-232, 1966.

[51] V. A.. Solonnikov. On general boundary problems for systems which are elliptic in the sense of A. Douglis and L. Nirenberg. II. Proc. Steklov Inst Math, 92:269-339, 1968.

[52] R. Temam. Navier-Stokes Equations. North-Holland, 1979. 